Supplementary Information to

\title{
The Role of Ferryl Ion Intermediates in Fast Fenton Chemistry on Aqueous Microdroplets
}

\author{
Alan Y. Gu1,2, Charles Musgrave², William A. Goddard III2, Michael R. Hoffmann, and Agustín J. \\ Colussi ${ }^{1 *}$ \\ ${ }^{1}$ Linde Laboratories, California Institute of Technology, Pasadena, California 91125, United States \\ ${ }^{2}$ Division of Chemistry and Chemical Engineering, California Institute of Technology, Pasadena, California 91125, \\ United States \\ *Towhom correspondence may be addressed:mrh@caltech.edu or ajcoluss@caltech.edu
}

This file includes:

Supplementary Notes

Online Electrospray Ionization Mass Spectrometry

Quantification of Ozone Concentration in Spray Chamber

Kinetic Analysis of $\mathrm{Me}_{2} \mathrm{SO}_{(\mathrm{aq})}$ Ozonation in Bulk Water

Supplementary Figures S1 to S15

Supplementary Tables S1 to S2

Supplementary List S1 


\section{Online Electrospray Ionization Mass Spectrometry}

In our gas-liquid experiments, the products generated in the microdroplets and the outermost layers of the microjets are analyzed without manipulation by online electrospray ionization mass spectrometry (ESI-MS). ${ }^{1,2}$ The production of charged microdroplets requires the conversion of the kinetic energy of the fast sheathing nebulizer $\mathrm{N}_{2}$ stream into electrostatic energy associated with the separation of anions from cations and that associated with the creation of additional surface. ${ }^{3}$ Charge separation is a direct result of the nebulizer gas because ion signals (1) increase at higher nebulizer gas velocity and (2) extrapolate to zero as nebulizer gas velocity drops to zero. ${ }^{2,3}$. The polarizing external electric field required to deflect the charged microdroplets does not affect the observed interfacial chemistry, as shown, e.g., in the interfacial $\alpha$-tocopherol ozonation reaction in the positive and negative modes. ${ }^{4}$ This is consistent with the fact that the calculated electric field at the microjet tip is 2 orders of magnitude weaker than the electric field naturally present at the air-water interface. ${ }^{5,6}$ The surface specificity of online ESI-MS has been demonstrated in several reports from our laboratory. ${ }^{1,7}$

\section{Quantification of Ozone Concentration in Spray Chamber}

Quantification of interfacial ozone concentration assumes a well-mixed spray chamber with an $\mathrm{O}_{3} / \mathrm{O}_{2(\mathrm{~g})}$ inlet at $50 \mathrm{~cm}^{3} / \mathrm{min}$ and a jet of nebulizer gas stream through an annulus with an inner diameter of 100 um and an outer diameter of 200 um at sonic velocity. Ozone generator settings at 1200, 2700 and $3000 \mathrm{ppm}$ in the $\mathrm{O}_{2} / \mathrm{O}_{3(\mathrm{~g})}$ stream correspond to 113,254 and $283 \mathrm{ppm}$ in the spray chamber, respectively.

\section{Kinetic Analysis of $\mathrm{Me}_{2} \mathrm{SO}_{(\mathrm{aq})}$ Ozonation in Bulk Water}

In this experiment, $\mathrm{Me}_{2} \mathrm{SO}_{(\mathrm{aq})}$ and appropriate internal standards are dissolved in an aqueous solution in syringe $A$, while saturated $\mathrm{O}_{3(\mathrm{aq})}$ aqueous solution in equilibrium with $3000 \mathrm{ppm}_{3(\mathrm{~g})}$ is prepared in syringe $B$. Syringe $A$ and $B$ are each pushed at $15 \mu \mathrm{L} / \mathrm{min}$ into a manifold tank reactor of volume $373 \mu \mathrm{L}$. The outlet of the manifold tank reactor connects to the ESI-MS microjet via PEEK capillary tubing of negligible internal volume.

Levanov et al. ${ }^{8}$ reported the Henry's Law constant for ozone $\mathrm{H}_{\mathrm{O}_{3}}$ as a function of temperature

$$
\begin{gathered}
\ln H_{O_{3}}=-(9.445)+\frac{2406}{T} \\
H_{O_{3}}=86.8 \text { at } 25^{\circ} \mathrm{C}
\end{gathered}
$$

Calculate the saturation concentration of $\mathrm{O}_{3}(\mathrm{aq})$ in equilibrium with $3000 \mathrm{ppm} \mathrm{O}_{3(\mathrm{~g})}$

$$
\left[O_{3 a q}\right]=0.0106 \mathrm{M}
$$


In the tank reactor, the concentration of $\mathrm{O}_{3}(\mathrm{aq})$ is diluted by half, resulting in an initial reaction concentration of

$$
\frac{\left[\mathrm{O}_{3_{a q}}\right]}{2}=0.00532 \mathrm{M}=255 \mathrm{mg} / \mathrm{L}
$$

At this ozone concentration, the ozonation $\mathrm{Me}_{2} \mathrm{SO}$ follows zero-order kinetics with a rate constant $\mathrm{k}=0.10 \mathrm{mM} / \mathrm{min}^{9}$

We can calculate this rate constant from our bulk-phase experimental measurements. From Figure $\mathrm{S} 3$, we obtain the $\mathrm{Me}_{2} \mathrm{SO}_{2}$ concentration from direct ozonation to be $2.335 \mathrm{mM}$. We calculate the reaction time using a continuously stirred tank reactor model with a total volume of $373 \mu \mathrm{L}$ and a total flow rate of $30 \mu \mathrm{L} / \mathrm{min}$, and the residence time is

$$
t=\frac{373}{30}=12.43 \mathrm{~min}
$$

Hence the zero-order rate constant is

$$
k_{0}=\frac{2.335}{12.43}=0.0188 \mathrm{mM} / \mathrm{min}
$$

Comparing with the literature reported value of $0.10 \mathrm{mM} / \mathrm{min},{ }^{9}$ this confirms the slow bulk phase kinetics compared to interfacial kinetics where $2 \mathrm{mM} \mathrm{Me}_{2} \mathrm{SO}_{2}$ are formed from $\mathrm{Me}_{2} \mathrm{SO}$ ozonolysis within $1 \mathrm{~ms}$ (Figure S11 y-intercept). 


\section{Figures}

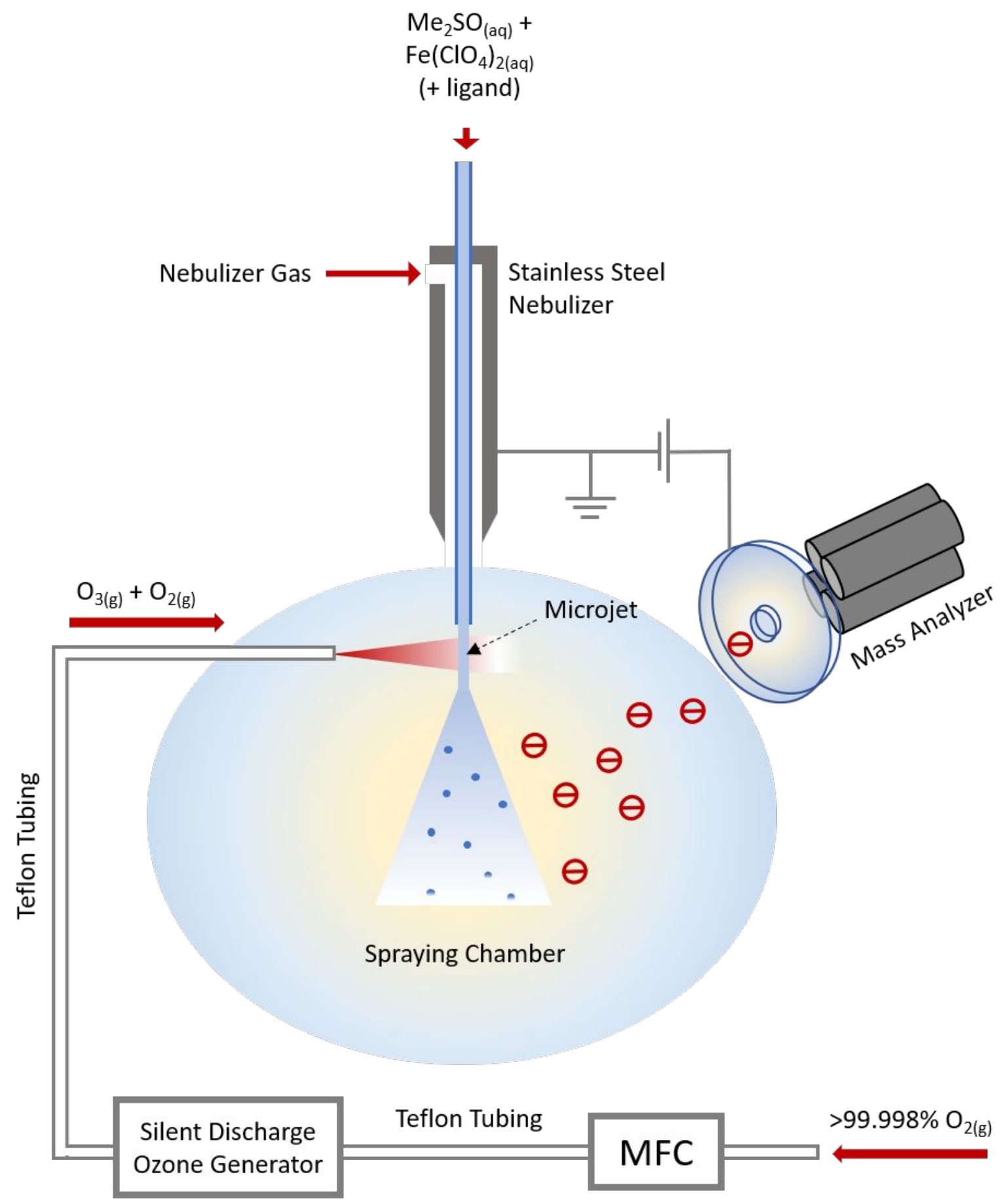

Figure S1. Experimental setup of online ES-MS. The aqueous reactants and gaseous reactants contact at the outlet tip of the microjet 


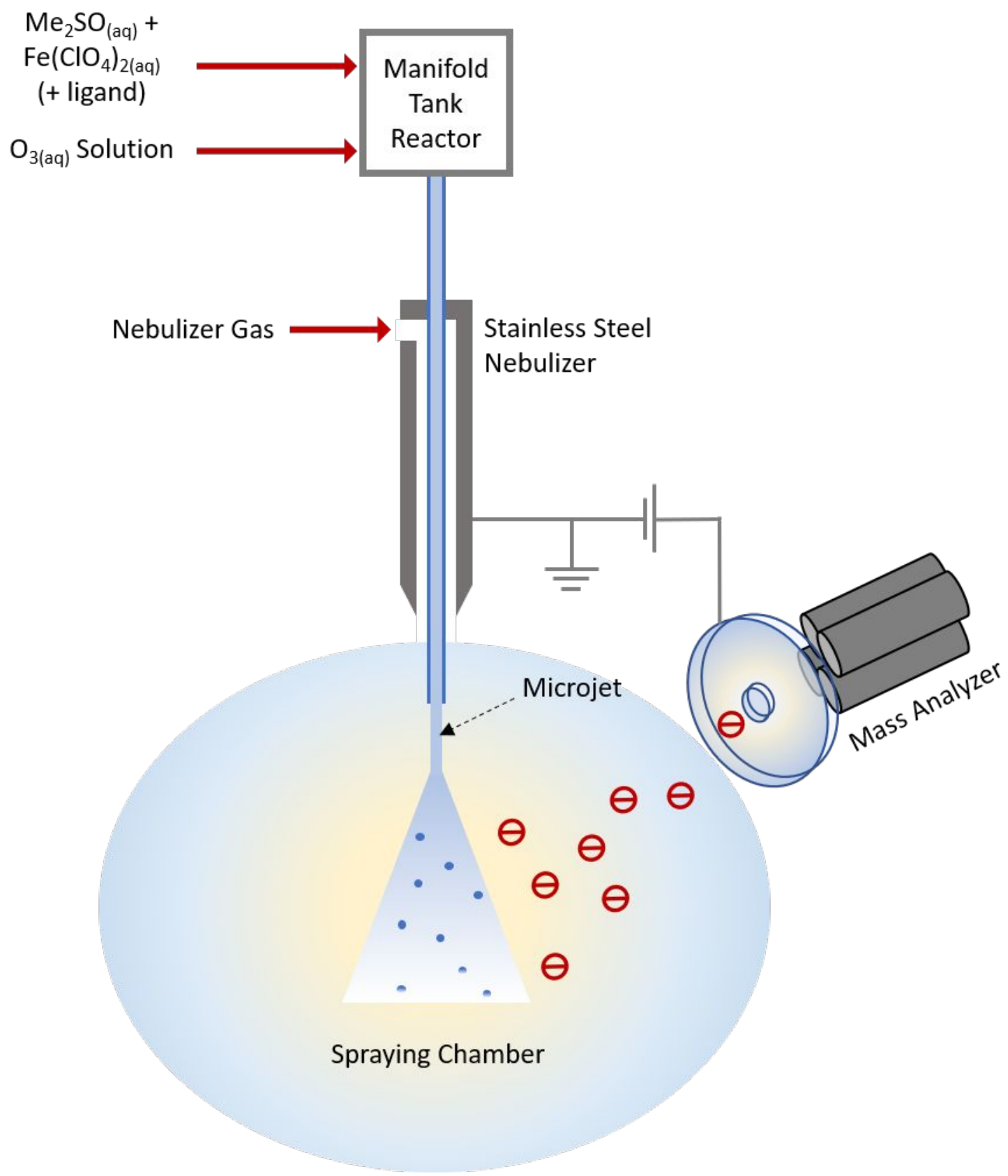

Figure S2. Experimental setup of offline ES-MS. The aqueous reactants contact each other in the manifold tank reactor before entering the microjet 


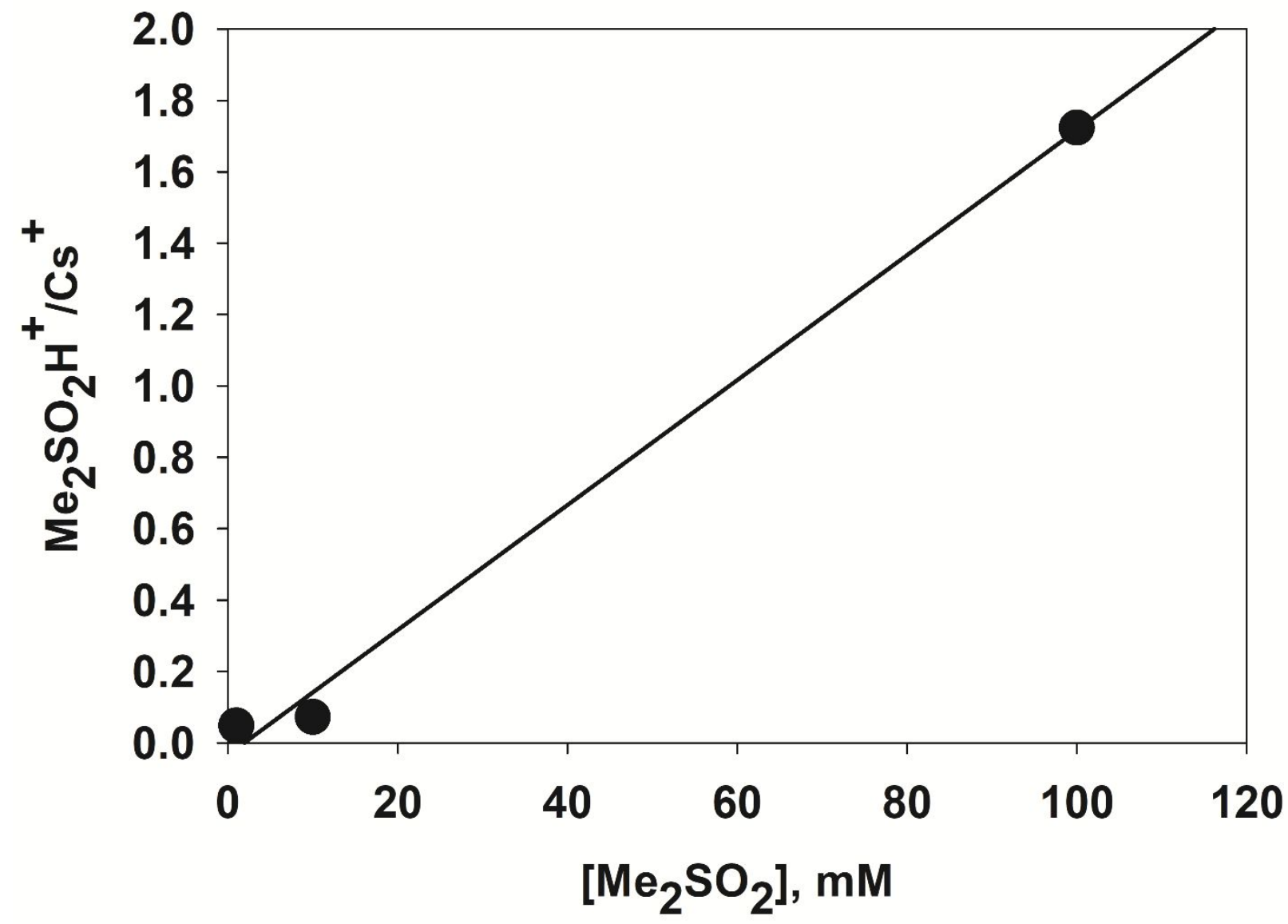

Figure S3. $\mathrm{Me}_{2} \mathrm{SO}_{2}$ calibration curve using 1, 10 and $100 \mathrm{mM} \mathrm{Me}_{2} \mathrm{SO}_{2}$. Y-axis plots the mass signal intensity ratios of $\mathrm{Me}_{2} \mathrm{SO}_{2} \mathrm{H}^{+} / \mathrm{Cs}^{+}$at $\mathrm{pH}=4.5$ and $\left[\mathrm{Cs}^{+}\right]=50 \mu \mathrm{M}$ 


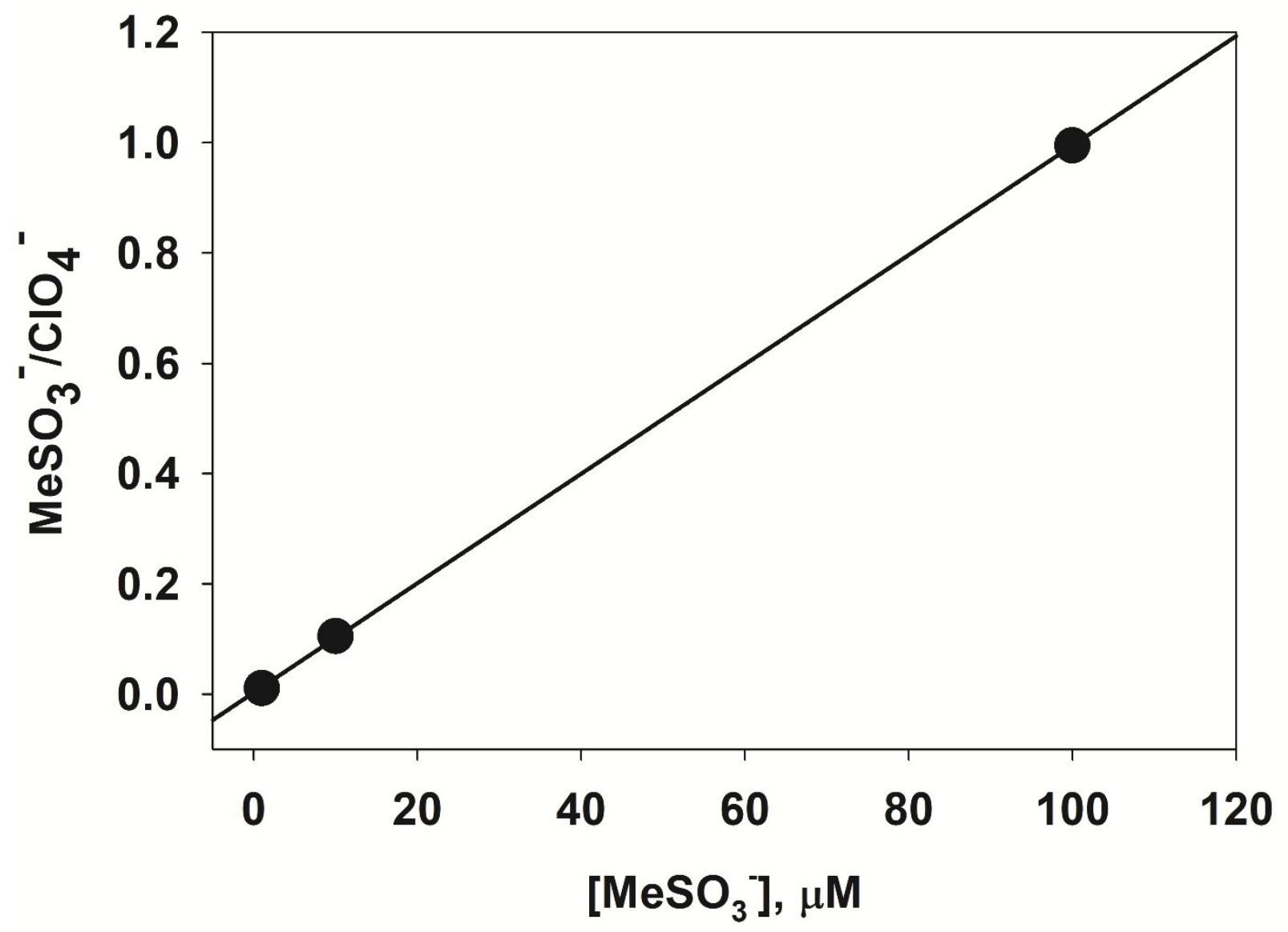

Figure S4. $\mathrm{MeSO}_{3}{ }^{-}$calibration curve using 1,10 and $100 \mu \mathrm{M} \mathrm{MeSO}{ }_{3} \mathrm{Na}$. Y-axis plots the mass signal intensity ratios of $\mathrm{MeSO}_{3}{ }^{-} / \mathrm{ClO}_{4}{ }^{-}$at $\mathrm{pH}=4.5$ and $\left[\mathrm{ClO}_{4}{ }^{-}\right]=250 \mu \mathrm{M}$ 


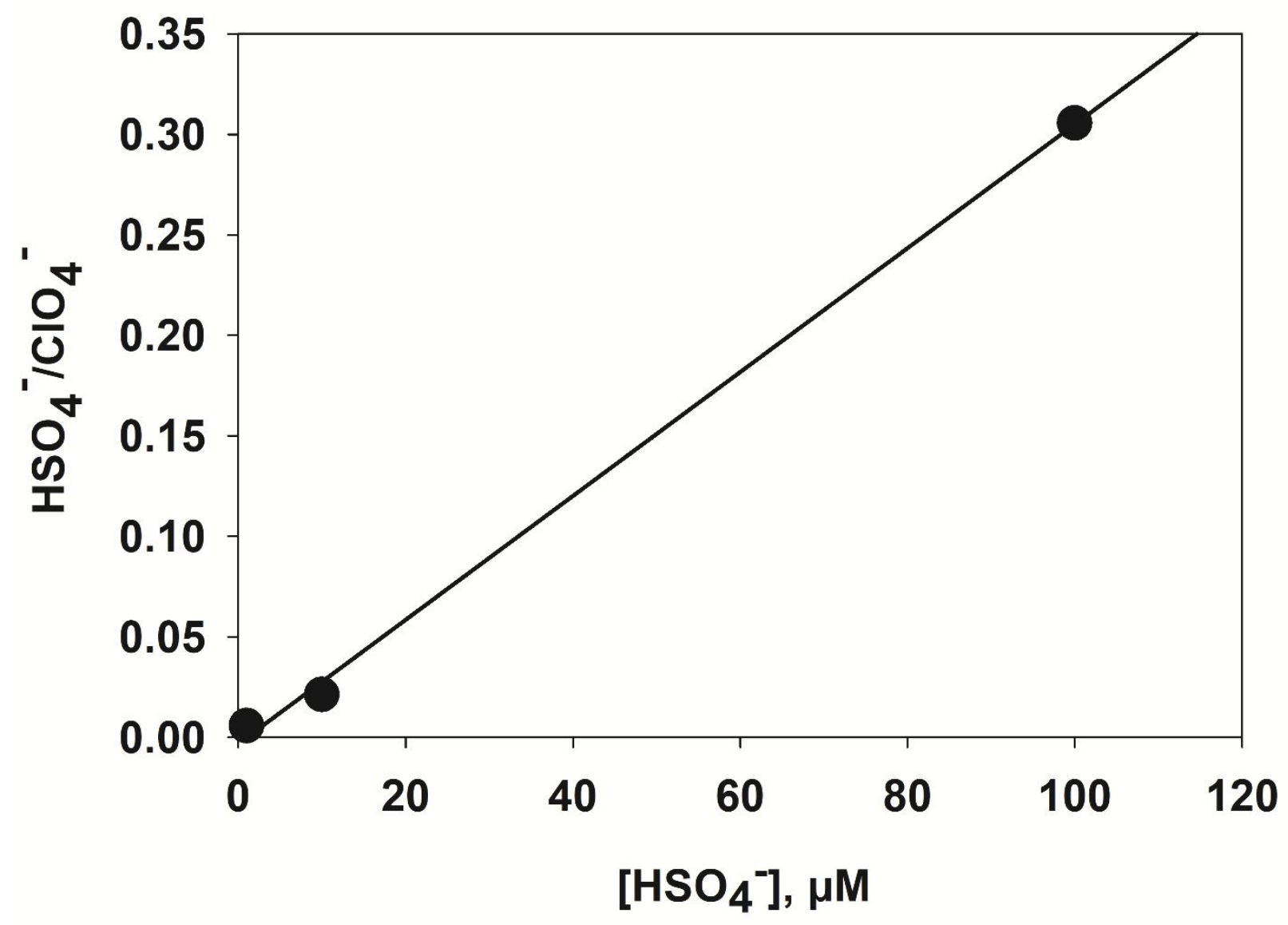

Figure S5. $\mathrm{HSO}_{4}^{-}$calibration curve using 1, 10 and $100 \mu \mathrm{M} \mathrm{NaHSO}_{4}$. Y-axis plots the mass signal intensity ratios of $\mathrm{HSO}_{4}^{-} / \mathrm{ClO}_{4}^{-}$at $\mathrm{pH}=4.5$ and $\left[\mathrm{ClO}_{4}^{-}\right]=250 \mu \mathrm{M}$ 
No Ligand:

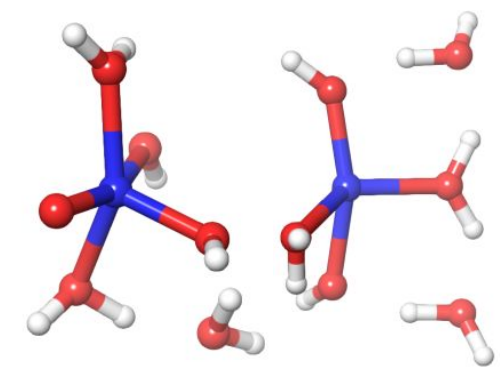

Dimethylamine:

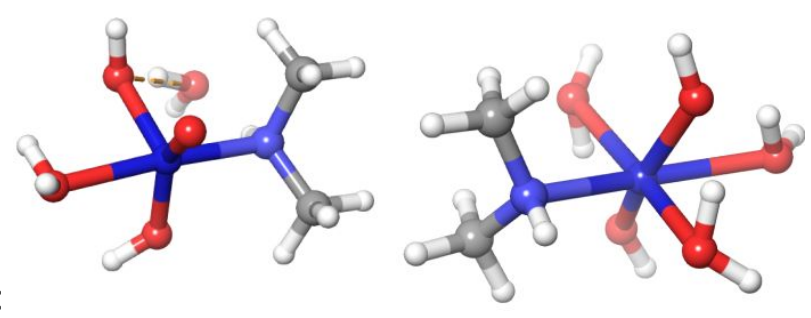

Trimethylamine:

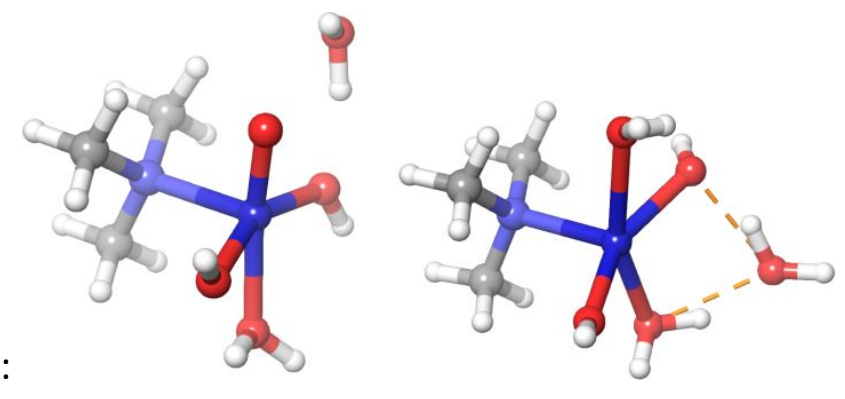

Chloride:

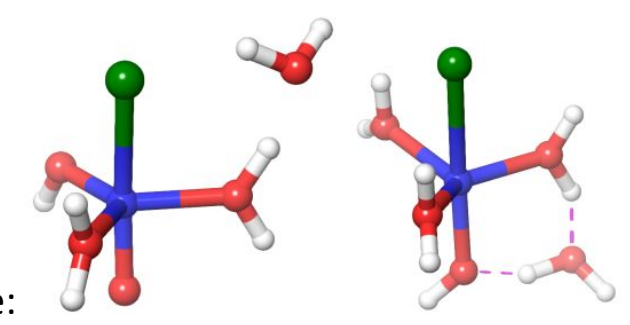

Oxalate:
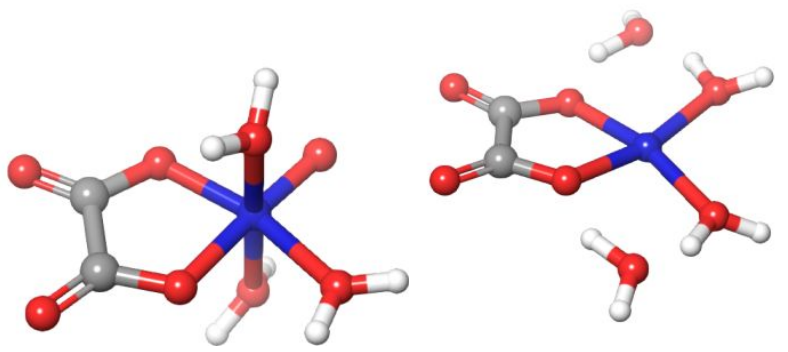

Malonate:
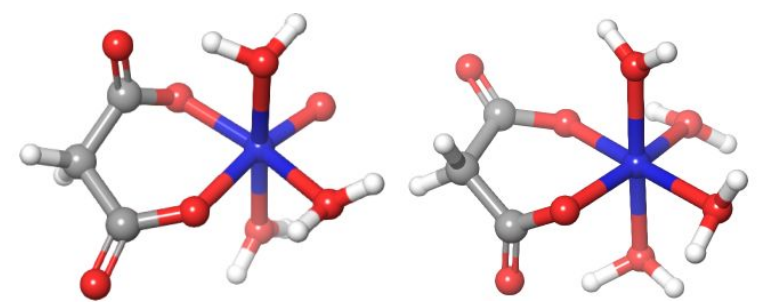
Tartrate:

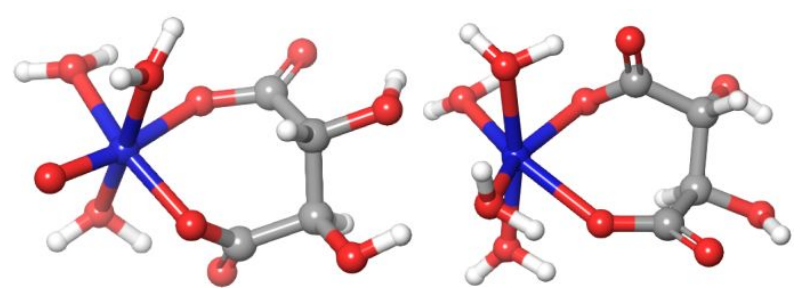

Catechol:
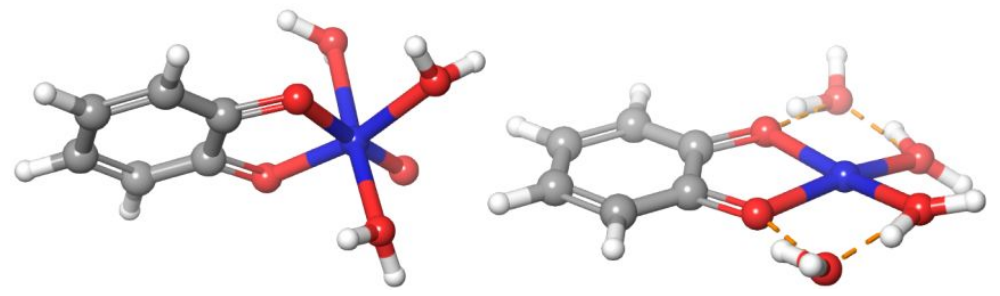

p-Benzoquinone:

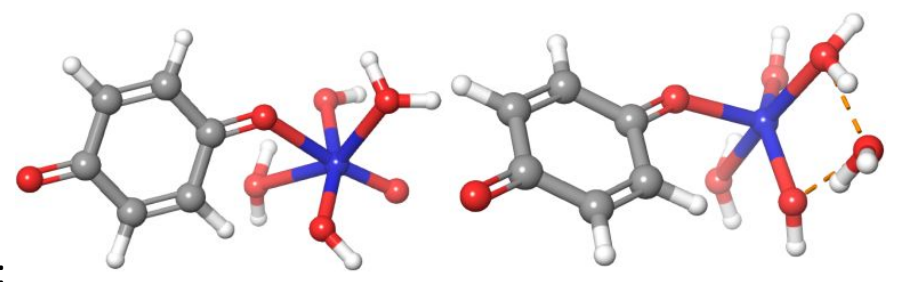

1,4-naphthaquinone:
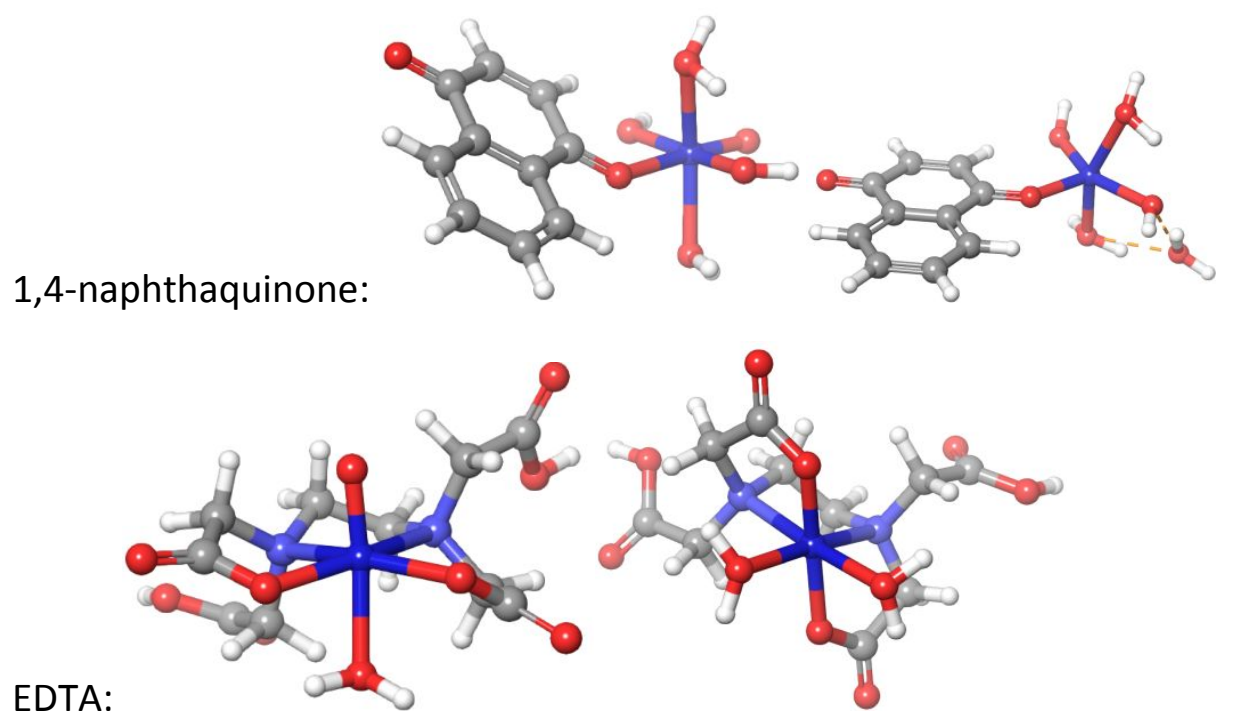

Figure S6. Optimized structures of ligated $\mathrm{Fe}^{2+}$ and $\mathrm{FeO}^{2+}$ complexes from DFT calculation 


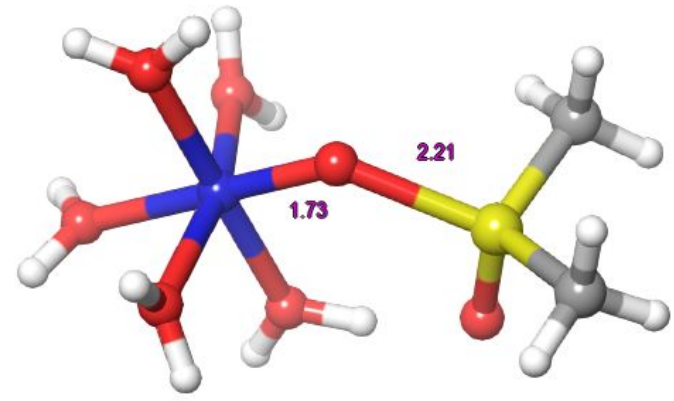

Figure S7. Optimized structure of $\mathbf{R} \mathbf{3}$ transition state 


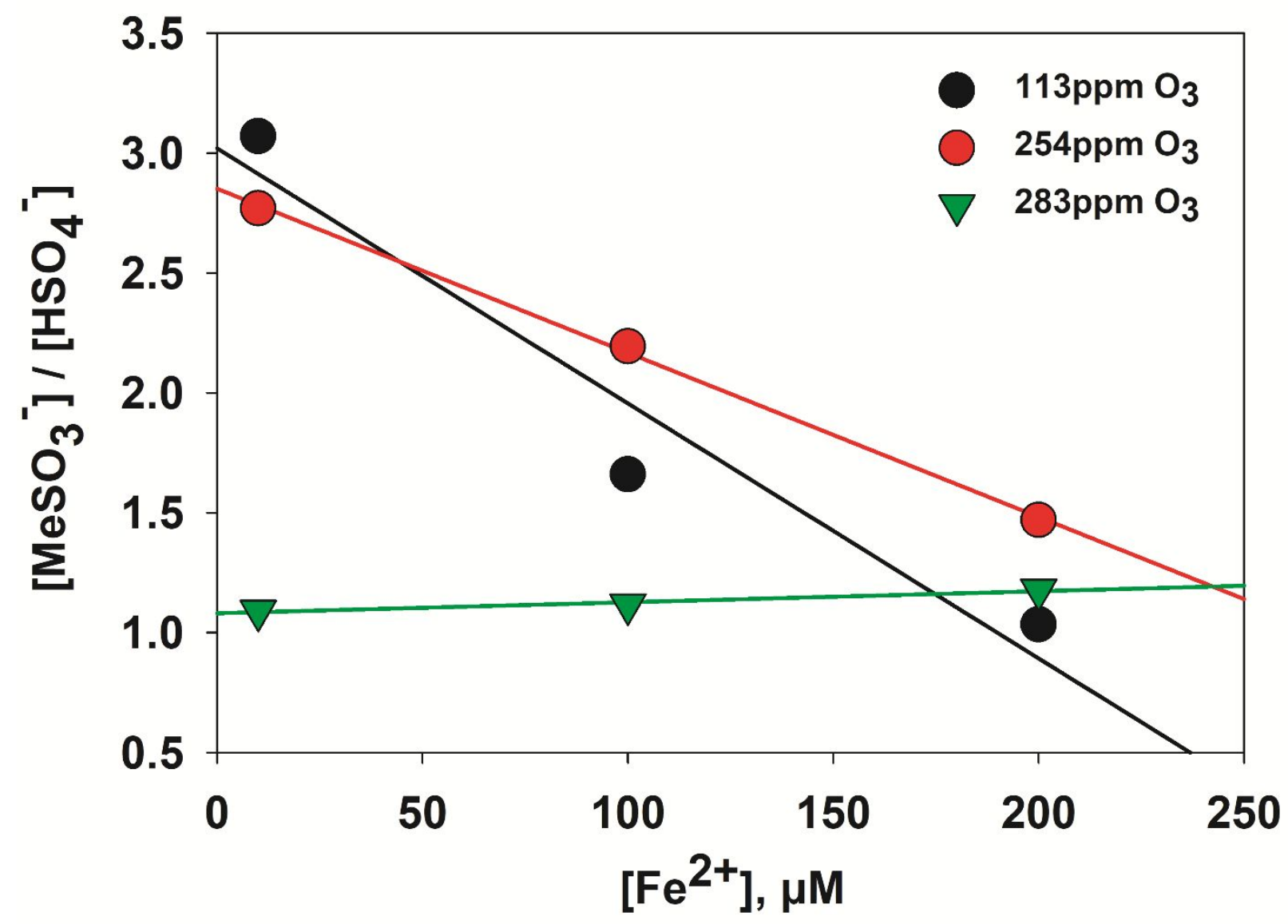

Figure S8. Relative product concentration of $\mathrm{MeSO}_{3}{ }^{-}$and $\mathrm{HSO}_{4}{ }^{-}$in interfacial ozone-Fenton chemistry for $1 \mathrm{mM} \mathrm{Me}_{2} \mathrm{SO}$ and $100 \mu \mathrm{M} \mathrm{Fe}\left(\mathrm{ClO}_{4}\right)_{2}$ 

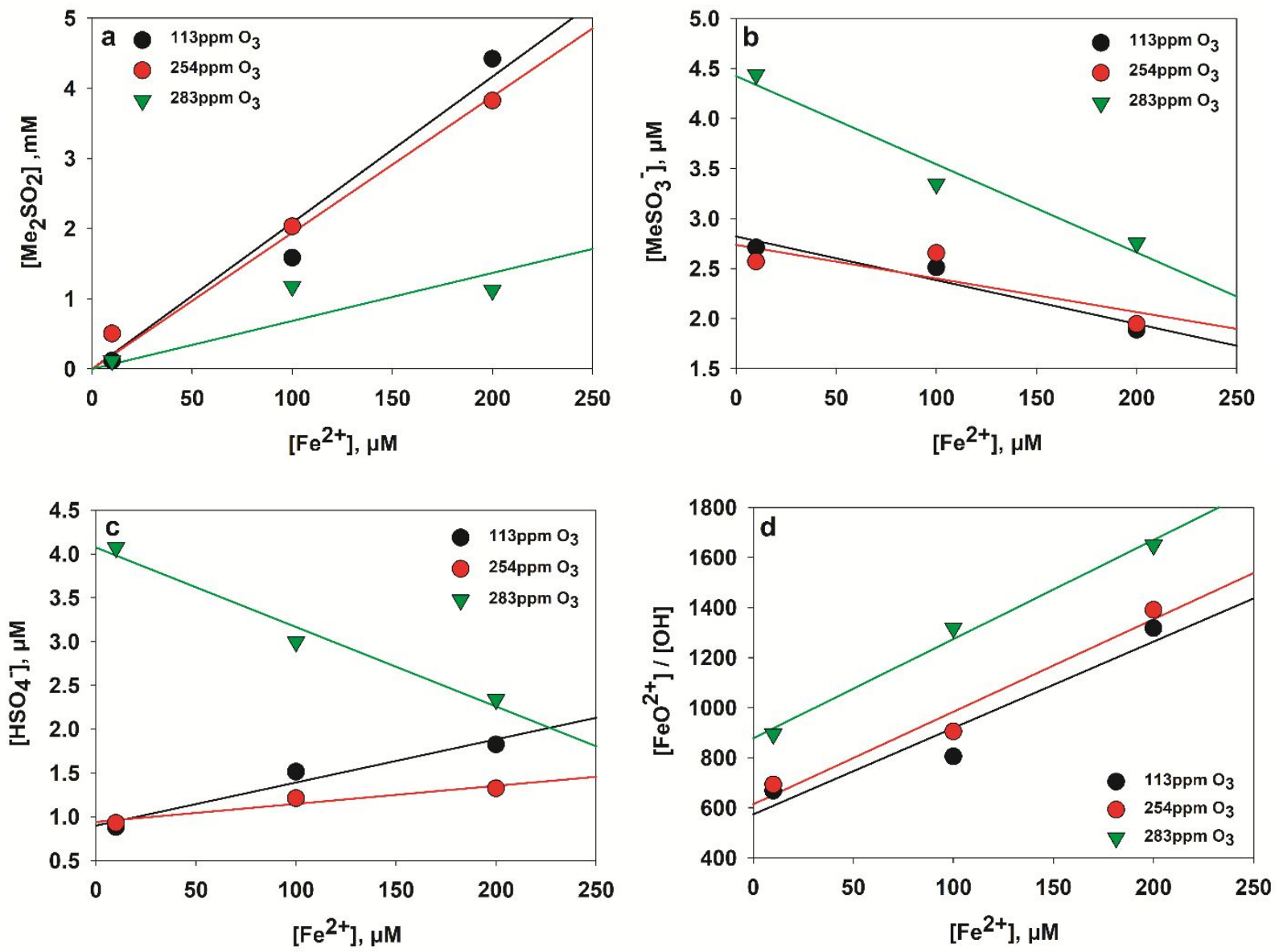

Figure S9. Product concentrations of (a) $\mathrm{Me}_{2} \mathrm{SO}_{2}$ (b) $\mathrm{MeSO}_{3}{ }^{-}$and (c) $\mathrm{HSO}_{4}{ }^{-}$at different initial Fe"l bulk concentration ([Fe"]) and gaseous $\mathrm{O}_{3}$ concentration at $\mathrm{pH} 4.5$ resulting from ozone Fenton oxidation of $1 \mathrm{mM} \mathrm{Me} \mathrm{MSO}_{2} \mathrm{SO}$. Concentrations on the $\mathrm{y}$-axis are the corresponding bulk concentrations of $\mathrm{Me}_{2} \mathrm{SO}_{2}, \mathrm{MeSO}_{3}$ - or $\mathrm{HSO}_{4}{ }^{-}$in hypothetical equilibrium with the sampled surface concentrations. The product concentrations are used to calculate the ferryl:hydroxyl pathway product concentrations ratio, represented as (d) the cumulative ferryl:hydroxyl intermediate. $\mathrm{CsClO}_{4}$ and $\mathrm{NaClO}_{4}$ are added as the internal standards to a total concentration of $50 \mu \mathrm{M} \mathrm{Cs}$ and $200 \mathrm{MM} \mathrm{ClO}_{4}^{-}$ 


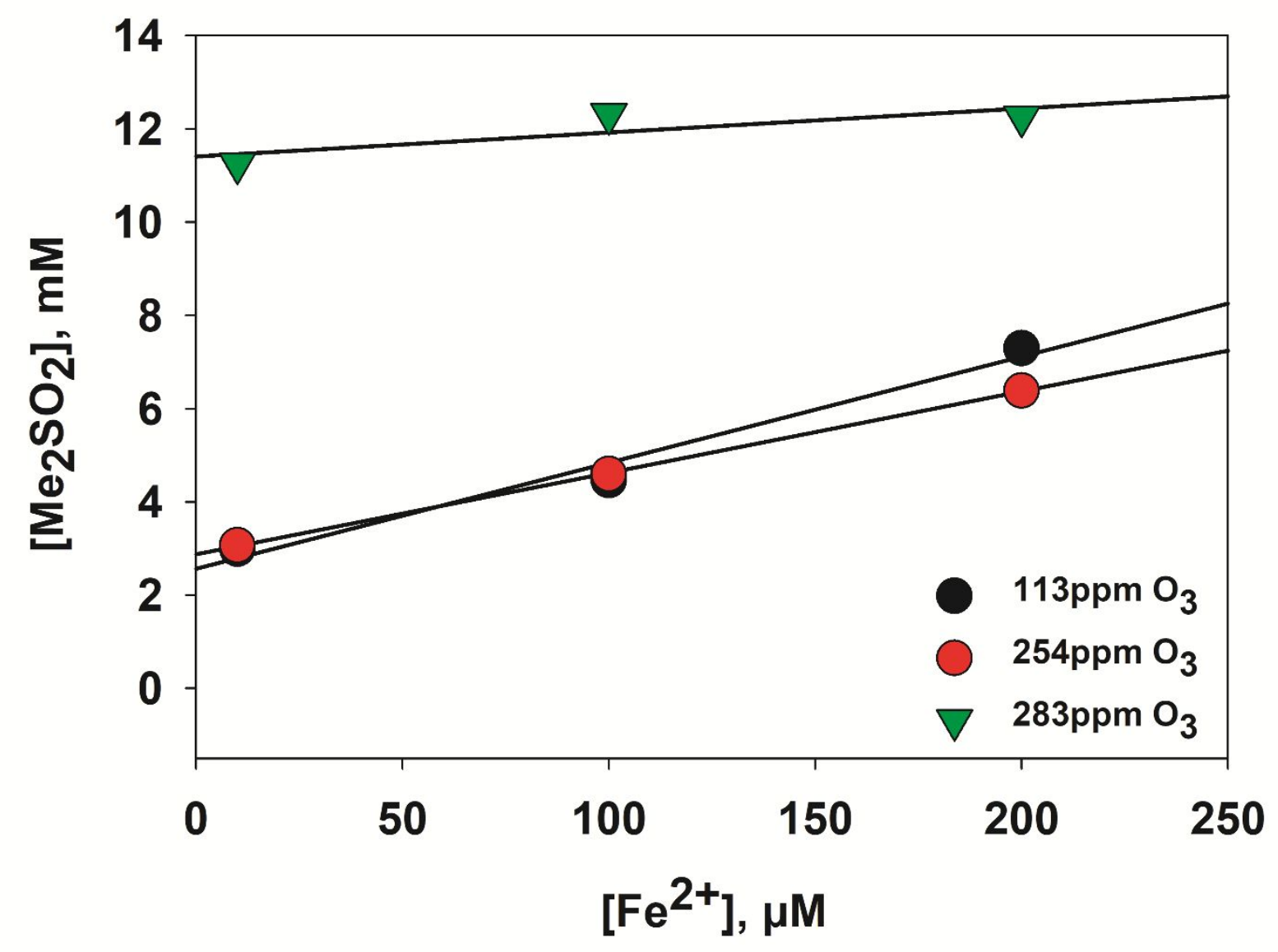

Figure S10. $\mathrm{Me}_{2} \mathrm{SO}_{2}$ product concentration at the air-water interface for $1 \mathrm{mM} \mathrm{Me}_{2} \mathrm{SO}$ and $100 \mu \mathrm{M} \mathrm{Fe}\left(\mathrm{ClO}_{4}\right)_{2}$ including $\mathrm{Me}_{2} \mathrm{SO}_{2}$ produced from $\mathrm{Me}_{2} \mathrm{SO}$ direct ozonolysis. The y-intercepts show the product concentration from direct ozonation of $\mathrm{Me}_{2} \mathrm{SO}$, and the slope shows the product concentration from ferryl O-atom transfer 


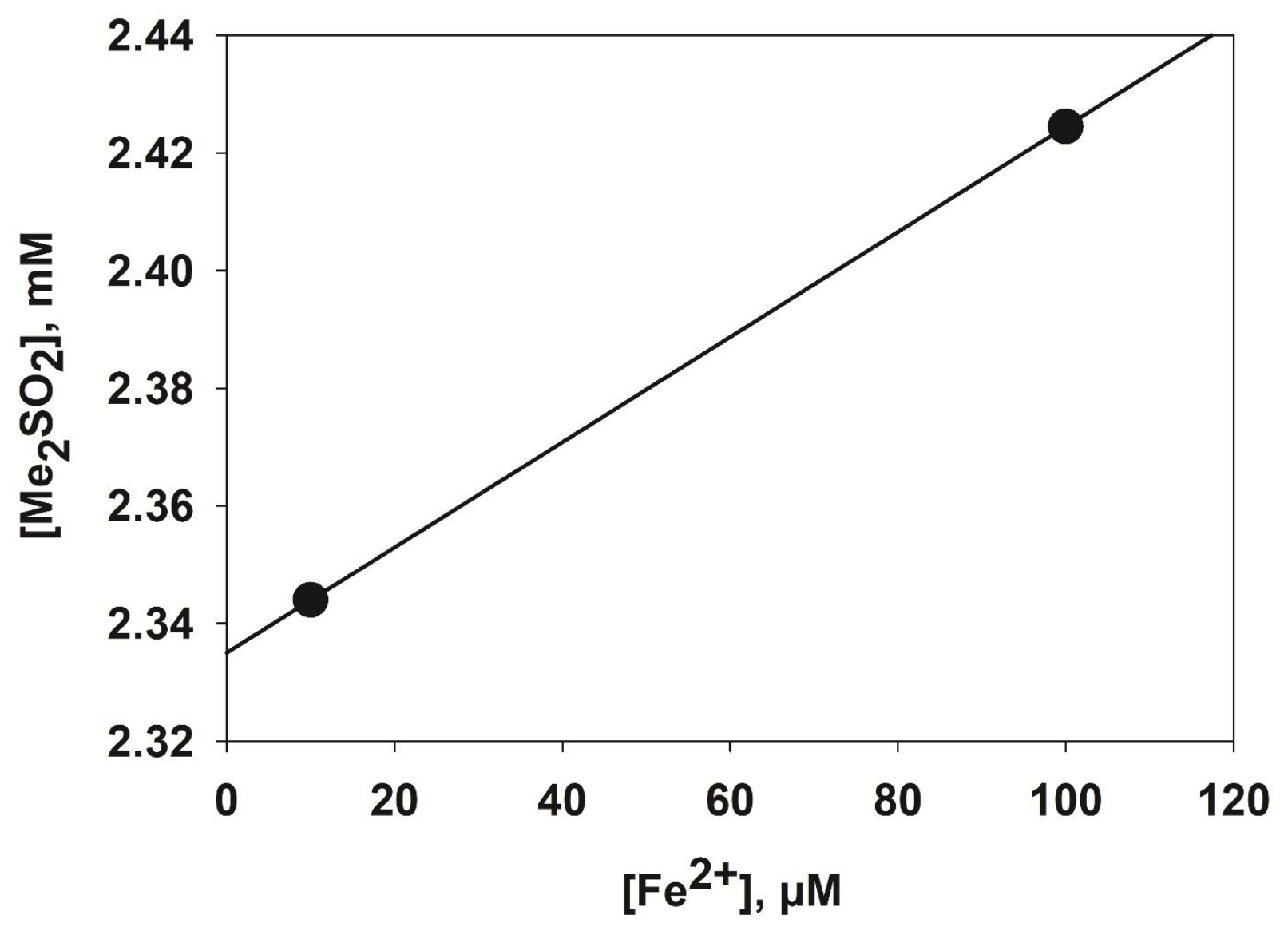

Figure S11. $\mathrm{Me}_{2} \mathrm{SO}_{2}$ product concentration in the bulk phase reaction from $10 \mathrm{mM} \mathrm{Me} \mathrm{SO}_{2}, 5 \mathrm{mM}$ $\mathrm{O}_{3}$ and $100 \mu \mathrm{M} \mathrm{Fe}\left(\mathrm{ClO}_{4}\right)_{2}$ reacting for 746 seconds, including $\mathrm{Me}_{2} \mathrm{SO}_{2}$ produced from $\mathrm{Me}_{2} \mathrm{SO}$ direct ozonolysis. The $y$-intercepts show the product concentration from direct ozonation of $\mathrm{Me}_{2} \mathrm{SO}$, and the slope shows the product concentration from ozone-Fenton chemistry, including but not limited to $\mathrm{O}$-atom transfer from ferryl species. The $\mathrm{Me}_{2} \mathrm{SO}_{2}$ product concentration originating from $100 \mu \mathrm{M} \mathrm{Fe}{ }^{2+}$ sets the upper bound for ferryl O-atom transfer kinetics 


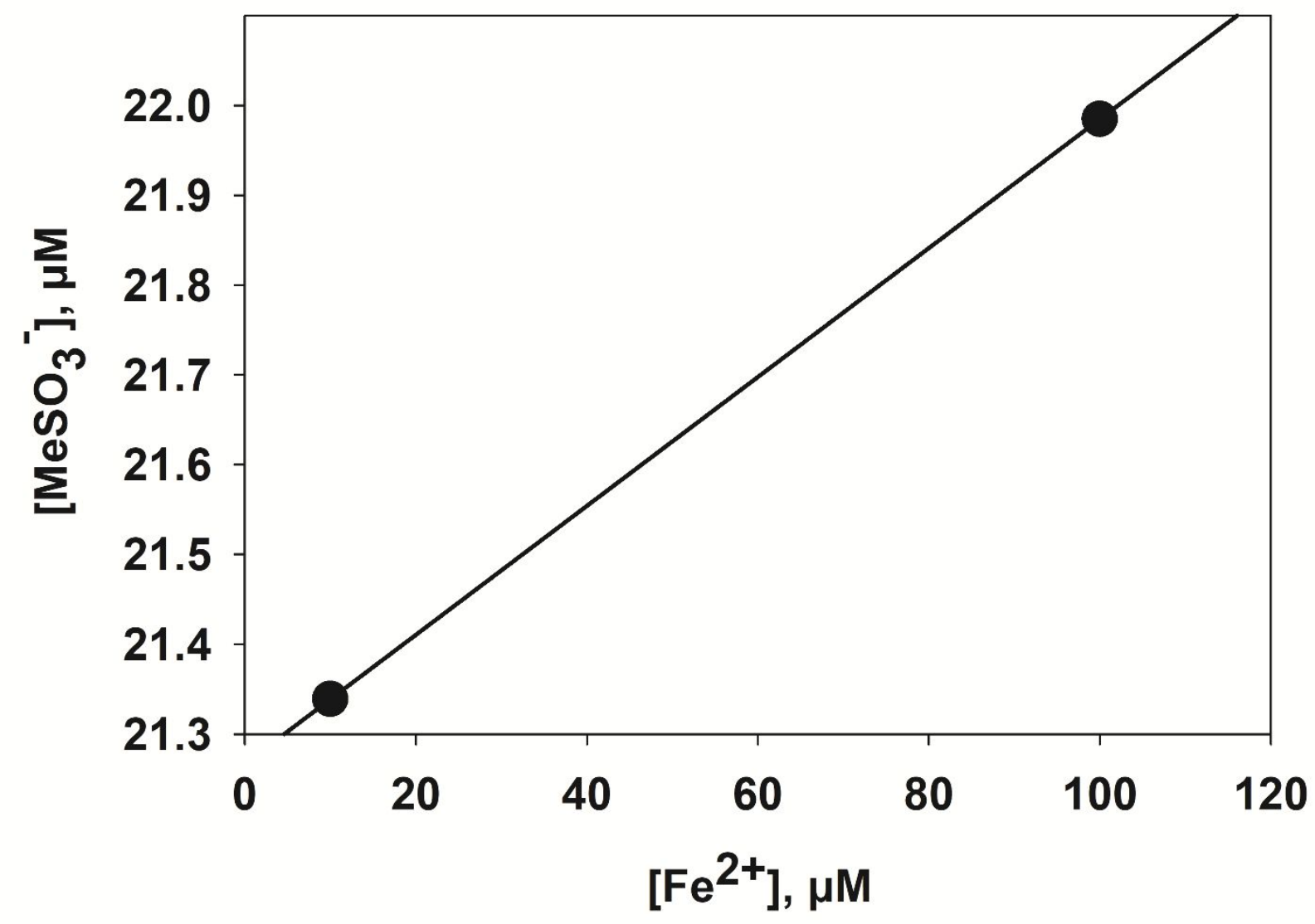

Figure S12. $\mathrm{MeSO}_{3}{ }^{-}$product concentration in bulk from $10 \mathrm{mM} \mathrm{Me}_{2} \mathrm{SO}, 5 \mathrm{mM} \mathrm{O}_{3}$ and $100 \mu \mathrm{M}$ $\mathrm{Fe}\left(\mathrm{ClO}_{4}\right)_{2}$ reacting for 746 seconds. The $y$-intercepts show the product concentration from direct ozonation of $\mathrm{Me}_{2} \mathrm{SO}$, and the slope shows the product concentration from ozone-Fenton chemistry, including both ferryl and $\mathrm{OH}$ pathways 


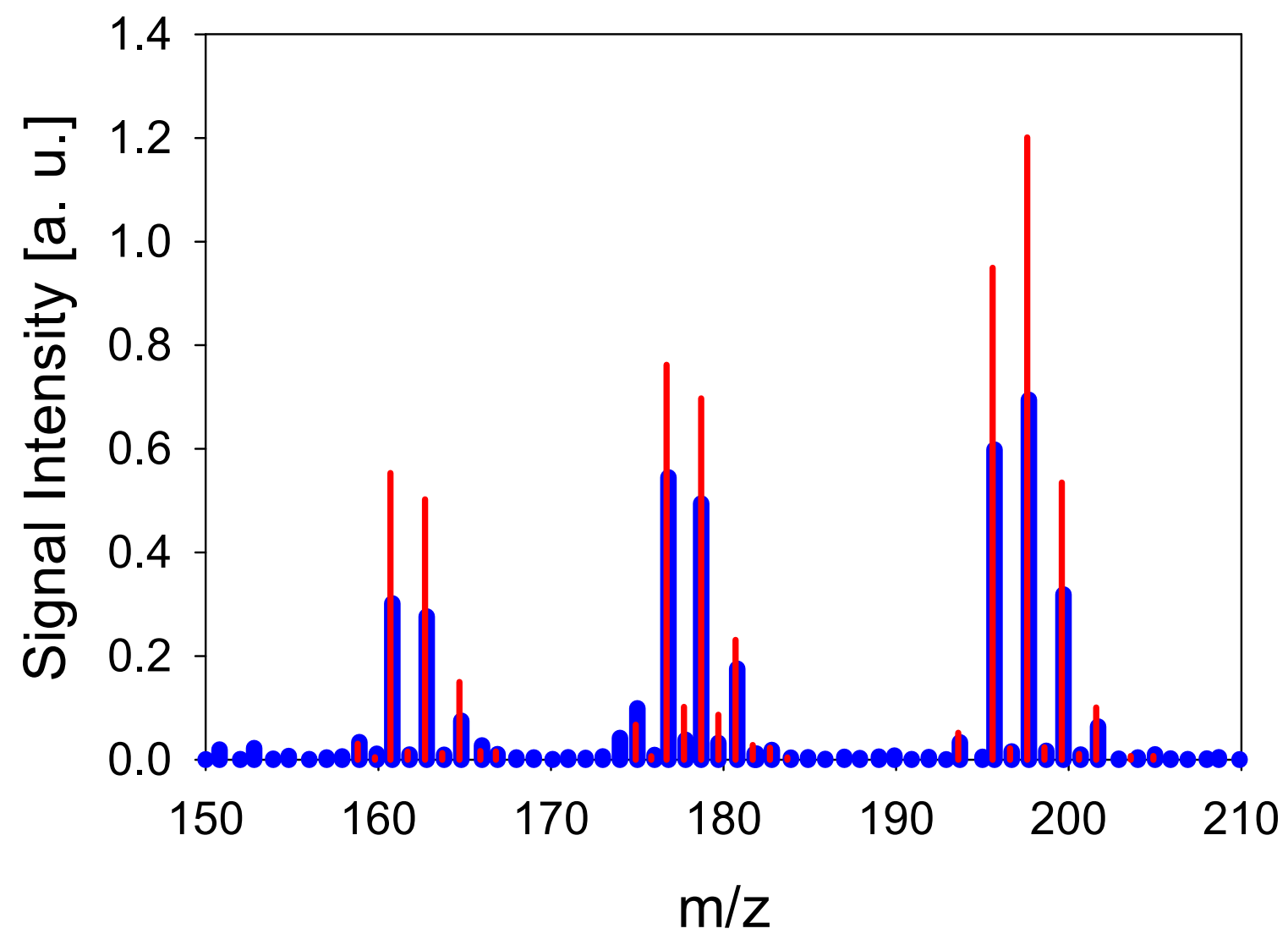

Figure S13. $5 \mathrm{mM}$ EDTA effect on the ozone Fenton chemistry between $10 \mathrm{mM} \mathrm{FeCl}_{2(\mathrm{aq})}$ and $283 p p m \mathrm{O}_{3(\mathrm{~g})}$. Blue denotes without EDTA and red with EDTA in the reaction mixture. The iron peaks are $\mathrm{Fe}{ }^{\prime \prime} \mathrm{Cl}_{3}{ }^{-}$most visible at $\mathrm{m} / \mathrm{z}=161 / 163 / 165, \mathrm{Fe}^{\mathrm{IV}} \mathrm{OCl}_{3}{ }^{-}$most visible at $\mathrm{m} / \mathrm{z}=177 / 179 / 181$, and $\mathrm{Fe}^{\mathrm{III}} \mathrm{Cl}_{4}{ }^{-}$most visible at $\mathrm{m} / \mathrm{z}=196 / 198 / 200 / 202$. The signal intensities are lowered for all three species with EDTA added, showing that ligands compete with $\mathrm{Cl}^{-}$for the coordination sphere of $\mathrm{Fe}^{2+}$ ions 


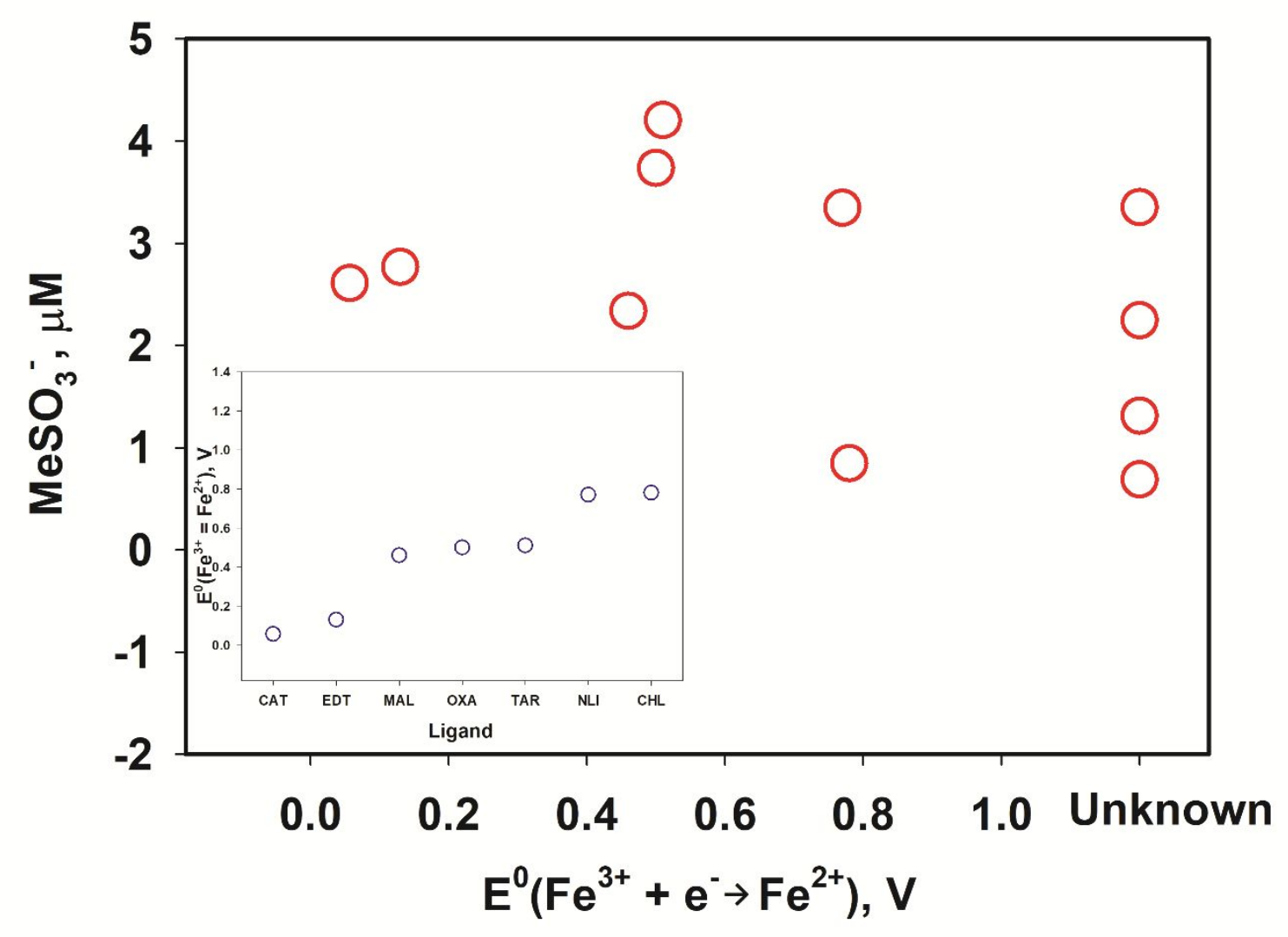

Figure S14. Methanesulfonate formation $\left[\mathrm{MeSO}_{3}^{-}\right.$] vs $\mathrm{E}^{0}\left(\mathrm{Fe}^{3+}+\mathrm{e}^{-} \longrightarrow \mathrm{Fe}^{2+}\right)$ reduction potentials. The inset shows calculated $\mathrm{E}^{0}\left(\mathrm{Fe}^{3+}+\mathrm{e}^{-} \longrightarrow \mathrm{Fe}^{2+}\right)$ potentials as functions of ligand. Standard reduction potentials of $\mathrm{Fe}^{3+}$ to $\mathrm{Fe}^{2+}$ in the presence of ligands shown on the $\mathrm{x}$-axis of the inset are from Martell and Smith. ${ }^{10}$ Reactants are $1 \mathrm{mM} \mathrm{Me}_{2} \mathrm{SO}, 100 \mu \mathrm{M} \mathrm{Fe}\left(\mathrm{ClO}_{4}\right)_{2}, 200 \mu \mathrm{M}$ ligand L, 283ppm $\mathrm{O}_{3}$ at the interface of gaseous $\mathrm{O}_{3}$ and aqueous microdroplets. Abbreviations: $\mathrm{DMA}=$ dimethylamine, $\mathrm{TMA}$ = trimethylamine, $\mathrm{CAT}$ = catechol, NAP = naphthoquinone, $\mathrm{NLI}$ = no ligand, $\mathrm{PBQ}=\mathrm{p}$-benzoquinone, $\mathrm{MAL}=$ malonate, $\mathrm{CHL}=$ chloride, $\mathrm{OXA}=$ oxalate, $\mathrm{TAR}=$ tartrate, $\mathrm{EDT}=$ ethylenediaminetetraacetate 


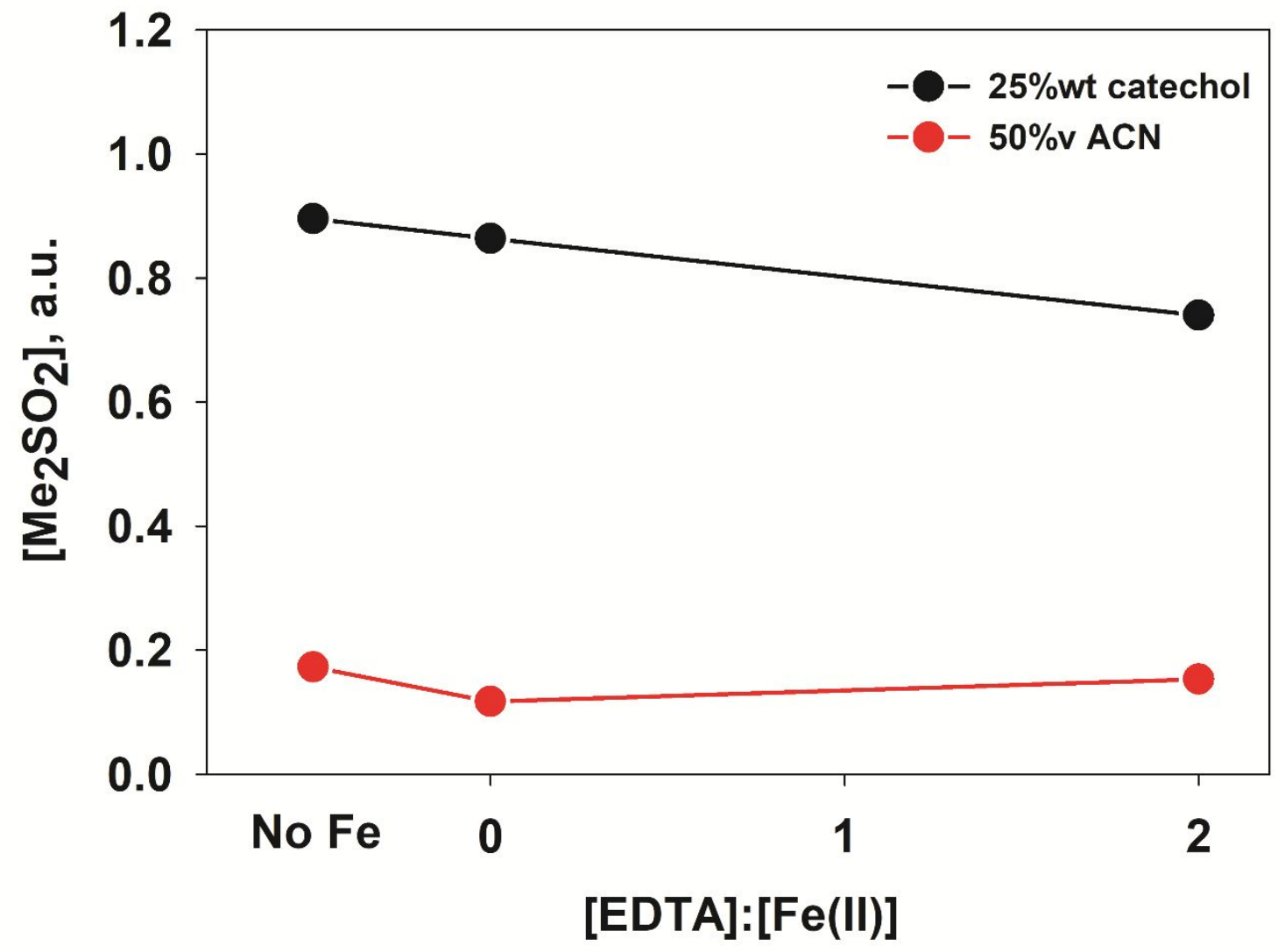

Figure S15. Ligand effect on interfacial $\mathrm{Me}_{2} \mathrm{SO}_{2}$ product concentration in 25 weight-percent catechol/water and 50 volume-percent acetonitrile/water solvents. The concentrations of $\mathrm{Me}_{2} \mathrm{SO}$ and $\mathrm{Fe}(\mathrm{II})$ are fixed at $1 \mathrm{mM}$ and $100 \mu \mathrm{M}$, respectively. $\mathrm{O}_{3(\mathrm{~g})}$ is at $283 \mathrm{ppm}$ 


\begin{tabular}{|c|c|}
\hline Ligand & $\mathrm{MeSO}_{3}{ }^{-} / \mathrm{HSO}_{4}^{-}$ \\
\hline catechol & 1.110045 \\
\hline EDTA & 3.297675 \\
\hline malonate & 1.774516 \\
\hline oxalate & 1.867809 \\
\hline tartrate & 1.966674 \\
\hline $\mathrm{ClO}_{4}^{-}$ & 1.116289 \\
\hline $\mathrm{Cl}^{-}$ & 15.35224 \\
\hline p-benzoquinone & 2.344543 \\
\hline 1,4-naphthaquinone & 0.86446 \\
\hline dimethylamine & 2.680139 \\
\hline trimethylamine & 0.758585 \\
\hline
\end{tabular}

Table S1. Ligand effect on the relative product concentrations of $\mathrm{MeSO}_{3}{ }^{-}$and $\mathrm{HSO}_{4}{ }^{-} \cdot \mathrm{ClO}_{4}{ }^{-}$ denotes no ligand as perchlorate does not ligate $\mathrm{Fe}^{2+}$ 


\begin{tabular}{|c|c|c|c|}
\hline Ligand & $\begin{array}{c}\mathrm{Fe}=0 \text { distance } \\
\text { (A) }\end{array}$ & $\begin{array}{l}\text { Fe(IV)-Ligand distance(s) } \\
\text { (A) }\end{array}$ & $\begin{array}{l}\text { Fe(II)-Ligand } \\
\text { distance(s) (Å) }\end{array}$ \\
\hline No Ligand & 1.65 & - & - \\
\hline Dimethylamine & 1.65 & 2.05 & 2.18 \\
\hline Trimethylamine & 1.64 & 2.17 & 2.17 \\
\hline Chloride & 1.64 & 2.43 & 2.63 \\
\hline Oxalate & 1.65 & $1.90,1.93$ & $1.98,1.99$ \\
\hline Malonate & 1.64 & $1.92,1.96$ & $1.92,1.96$ \\
\hline Tartrate & 1.64 & $1.92,1.96$ & $2.08,2.23$ \\
\hline Catechol & 1.66 & $1.89,1.91$ & $1.98,1.99$ \\
\hline p-benzoquinone & 1.65 & 2.16 & 2.01 \\
\hline 1,4-naphthaquinone & 1.65 & 2.12 & 2.03 \\
\hline EDTA & 1.63 & $\begin{array}{l}\text { Fe-N: } 2.26,2.33 \\
\text { Fe-O: } 1.95,1.96\end{array}$ & $\begin{array}{l}\text { Fe-N: } 2.20,2.22 \\
\text { Fe-O: } 1.91,1.90\end{array}$ \\
\hline
\end{tabular}

Table S2. Important bond distances for the Fe systems investigated. All distances are in $\AA$. Some structures contain multiple Fe-Ligand distances due to increased denticity. Structures are depicted in Figure 4 


\section{Lists}

\begin{tabular}{|c|c|c|c|}
\hline \multicolumn{4}{|c|}{ no ligand } \\
\hline $\mathrm{O} 14$ & 0.7680228217 & -1.3052848462 & -2.2919432579 \\
\hline $\mathrm{H} 2$ & 0.7584223414 & -2.2501763863 & -2.0661375355 \\
\hline $\mathrm{Fe} 4$ & -0.7497366749 & -0.0067991904 & -2.0394507429 \\
\hline O5 & -1.0603443397 & 0.3322663998 & -4.0031504829 \\
\hline H19 & -0.4036576990 & 0.0111010687 & -4.6772658472 \\
\hline $\mathrm{H} 20$ & -1.9243029173 & 0.7630532931 & -4.2591255829 \\
\hline O9 & -2.3928455969 & 0.6745027680 & -1.3910942292 \\
\hline $\mathrm{H} 23$ & -2.7752923342 & 0.7883218354 & -0.5090241509 \\
\hline $\mathrm{O} 10$ & -3.3577077614 & 1.4137914804 & -3.6805626934 \\
\hline H11 & -4.2761494874 & 1.2317566774 & -3.9309555931 \\
\hline $\mathrm{H} 12$ & -3.1649396027 & 1.2110512342 & -2.6989924854 \\
\hline $\mathrm{O} 25$ & 0.8722547616 & 0.7285103625 & -0.8588231959 \\
\hline $\mathrm{H} 29$ & 1.4614618184 & -0.0136329128 & -1.1579825200 \\
\hline H30 & 1.3011820145 & 1.5741223300 & -0.6654673386 \\
\hline $\mathrm{O} 15$ & 1.0136826305 & -0.8901396470 & -4.8566046002 \\
\hline H16 & 1.8627748015 & -0.8113791205 & -5.3154377222 \\
\hline H17 & 1.0934667770 & -1.1445303650 & -3.8734238435 \\
\hline dma-I & & & \\
\hline $\mathrm{O} 14$ & 0.7680228217 & -1.3052848462 & -2.2919432579 \\
\hline $\mathrm{H} 2$ & 0.7584223414 & -2.2501763863 & -2.0661375355 \\
\hline $\mathrm{Fe} 4$ & -0.7497366749 & -0.0067991904 & -2.0394507429 \\
\hline O5 & -1.0603443397 & 0.3322663998 & -4.0031504829 \\
\hline H19 & -0.4036576990 & 0.0111010687 & -4.6772658472 \\
\hline $\mathrm{H} 20$ & -1.9243029173 & 0.7630532931 & -4.2591255829 \\
\hline O9 & -2.3928455969 & 0.6745027680 & -1.3910942292 \\
\hline $\mathrm{H} 23$ & -2.7752923342 & 0.7883218354 & -0.5090241509 \\
\hline $\mathrm{O} 10$ & -3.3577077614 & 1.4137914804 & -3.6805626934 \\
\hline H11 & -4.2761494874 & 1.2317566774 & -3.9309555931 \\
\hline H12 & -3.1649396027 & 1.2110512342 & -2.6989924854 \\
\hline $\mathrm{O} 25$ & 0.8722547616 & 0.7285103625 & -0.8588231959 \\
\hline $\mathrm{H} 29$ & 1.4614618184 & -0.0136329128 & -1.1579825200 \\
\hline $\mathrm{H} 30$ & 1.3011820145 & 1.5741223300 & -0.6654673386 \\
\hline O15 & 1.0136826305 & -0.8901396470 & -4.8566046002 \\
\hline H16 & 1.8627748015 & -0.8113791205 & -5.3154377222 \\
\hline H17 & 1.0934667770 & -1.1445303650 & -3.8734238435 \\
\hline tma-F & & & \\
\hline $\mathrm{O} 14$ & -0.1655340093 & -1.7268063039 & -2.6095353944 \\
\hline $\mathrm{H} 2$ & -0.3944219122 & -2.4108828075 & -3.2632278177 \\
\hline $\mathrm{Fe} 4$ & -0.7707577602 & 0.2290541593 & -2.7723227762 \\
\hline O5 & 1.5230329615 & 0.0171089392 & -3.2915139341 \\
\hline H19 & 2.2534865858 & 0.4255137552 & -2.7999395253 \\
\hline $\mathrm{H} 20$ & 1.2970082143 & -0.9133447146 & -2.9833107091 \\
\hline O9 & -1.0066552088 & 1.9487382218 & -2.0424117983 \\
\hline $\mathrm{H} 23$ & -0.4984929168 & 2.6898021231 & -1.6832136443 \\
\hline $\mathrm{O} 10$ & -2.7446668203 & -0.2142757076 & -1.8892807656 \\
\hline H11 & -2.6311698838 & -0.9745628100 & -1.2442971945 \\
\hline H12 & -2.9093194947 & 0.6370485030 & -1.4418975663 \\
\hline $\mathrm{C} 15$ & -0.3704657242 & 1.5432800780 & -5.3973751225 \\
\hline N16 & -1.3494873767 & 0.5781975649 & -4.8293540290 \\
\hline $\mathrm{C} 17$ & -1.3520134653 & -0.6929262087 & -5.5942865208 \\
\hline $\mathrm{C} 18$ & -2.7126537661 & 1.1736537290 & -4.7711098241 \\
\hline $\mathrm{H} 17$ & -0.3698260792 & 2.4343964075 & -4.7735754166 \\
\hline H18 & 0.6210145965 & 1.0993010209 & -5.3613931753 \\
\hline $\mathrm{H} 21$ & -0.6314231817 & 1.8119699517 & -6.4290141523 \\
\hline $\mathrm{H} 22$ & -3.3882196531 & 0.4632592938 & -4.3008771379 \\
\hline $\mathrm{H} 24$ & -2.6684113541 & 2.0624700457 & -4.1458264821 \\
\hline $\mathrm{H} 25$ & -3.0734805126 & 1.4336554283 & -5.7743633641 \\
\hline $\mathrm{H} 26$ & -2.0433699061 & -1.3905771743 & -5.1239071940 \\
\hline $\mathrm{H} 27$ & -1.6590481908 & -0.5333790764 & -6.6357488419 \\
\hline
\end{tabular}




\begin{tabular}{|c|c|c|c|}
\hline $\mathrm{H} 28$ & -0.3506032286 & -1.1197469178 & -5.5796786368 \\
\hline $\mathrm{O} 25$ & -1.7216096955 & -2.2138716360 & -0.6271053384 \\
\hline $\mathrm{H} 29$ & -0.9584423562 & -2.1602443259 & -1.3060684009 \\
\hline $\mathrm{H} 30$ & -1.5271258971 & -2.7088429317 & 0.1810021166 \\
\hline \multicolumn{4}{|c|}{ chloride-Fe(II) } \\
\hline O14 & 0.3976702798 & -1.4773996919 & -2.7887423748 \\
\hline $\mathrm{H} 2$ & 0.3772259047 & -2.3828298976 & -2.4402323102 \\
\hline $\mathrm{Fe} 4$ & -0.7744413237 & 0.0396878672 & -2.3567048422 \\
\hline O5 & -0.4405337329 & 0.9590007201 & -4.1347828722 \\
\hline H19 & 0.2048408237 & 0.4563172448 & -4.7563223266 \\
\hline $\mathrm{H} 20$ & -0.7042765410 & 1.8602575824 & -4.3663319178 \\
\hline $\mathrm{Cl} 7$ & -2.0465718251 & 2.1906876266 & -1.5487949815 \\
\hline $\mathrm{O} 10$ & -2.7698035681 & -0.5746316041 & -2.2415785220 \\
\hline H11 & -3.2845621037 & -1.3024474873 & -2.6210595601 \\
\hline $\mathrm{H} 12$ & -3.2955943711 & 0.1981029804 & -1.9355223327 \\
\hline $\mathrm{O} 25$ & 0.0677119898 & 0.4013039493 & -0.4685131437 \\
\hline $\mathrm{H} 29$ & 0.9083819222 & 0.1111906487 & -0.0839362415 \\
\hline $\mathrm{H} 30$ & -0.3260204012 & 1.2087914841 & -0.0767820033 \\
\hline $\mathrm{O} 15$ & 1.1076792753 & -0.7458040964 & -5.0658618888 \\
\hline H15 & 2.0233219486 & -0.7675916562 & -5.3795276228 \\
\hline H16 & 0.9471859164 & -1.2229291780 & -4.1439162864 \\
\hline \multicolumn{4}{|c|}{ oxolate-Fe(II) } \\
\hline $\mathrm{C} 1$ & -1.3518626782 & 1.1887077594 & -4.9834823855 \\
\hline $\mathrm{C} 2$ & -2.5532196878 & 1.7486002828 & -4.1258181644 \\
\hline $\mathrm{O} 3$ & -0.5120180158 & 0.4353111114 & -4.2416854379 \\
\hline $\mathrm{O} 4$ & -2.4781394429 & 1.3550388065 & -2.8363637288 \\
\hline O5 & -3.4346454728 & 2.4545424885 & -4.6020014847 \\
\hline O6 & -1.2233866228 & 1.4270608016 & -6.1786955916 \\
\hline $\mathrm{Fe} 12$ & -0.9645174245 & 0.2308612041 & -2.3625262650 \\
\hline $\mathrm{O} 13$ & 0.7756771704 & -0.8846087072 & -2.4678183860 \\
\hline H14 & 1.4092866072 & -1.4248819202 & -1.9739703789 \\
\hline H15 & 0.9923498594 & -0.7718231176 & -3.4209800224 \\
\hline $\mathrm{O} 19$ & -1.9321294166 & 0.3648460913 & -0.5381685054 \\
\hline $\mathrm{H} 20$ & -1.9192305335 & 0.1031588534 & 0.3936904919 \\
\hline $\mathrm{H} 21$ & -2.6949722511 & 0.9318662328 & -0.7925921136 \\
\hline \multicolumn{4}{|c|}{ malonate-Fe(II) } \\
\hline $\mathrm{C} 1$ & -1.4616918542 & 1.1304608514 & -5.1661571065 \\
\hline $\mathrm{C} 2$ & -1.2714329443 & 2.5023583365 & -4.5030376946 \\
\hline $\mathrm{O} 3$ & -1.0968863375 & 0.0667678130 & -4.4235751927 \\
\hline $\mathrm{C} 4$ & -1.8047285398 & 2.6919085695 & -3.0755316379 \\
\hline H5 & -0.1931553953 & 2.6889511511 & -4.4657409888 \\
\hline O6 & -1.8804805410 & 1.0237516653 & -6.3236382582 \\
\hline $\mathrm{O} 14$ & -0.8041295636 & -2.0812953667 & -3.1379597763 \\
\hline $\mathrm{H} 2$ & -0.7242041158 & -3.0082010414 & -2.8728350970 \\
\hline $\mathrm{H} 3$ & -0.8822713734 & -1.9276317995 & -4.1088390855 \\
\hline $\mathrm{Fe} 4$ & -1.0391265617 & -0.1163462224 & -2.5090022568 \\
\hline O9 & -1.2111909422 & -0.0657317395 & -0.4407086955 \\
\hline $\mathrm{H} 23$ & -1.1927156691 & -0.5752420184 & 0.3813569757 \\
\hline H11 & -1.4663830043 & 0.8814262787 & -0.3420113948 \\
\hline $\mathrm{H} 21$ & -1.7252450582 & 3.2534853623 & -5.1385035080 \\
\hline $\mathrm{O} 21$ & -2.4638230784 & 3.6874660982 & -2.7578290328 \\
\hline $\mathrm{O} 23$ & -1.4646765589 & 1.7322976415 & -2.1928647874 \\
\hline \multicolumn{4}{|c|}{ tartrate-Fe(II) } \\
\hline O14 & -1.6514421105 & -1.8037446986 & -3.2016132395 \\
\hline $\mathrm{H} 2$ & -0.8782166179 & -2.3375215406 & -3.4554012886 \\
\hline H3 & -2.0250784525 & -1.2961771971 & -3.9689215532 \\
\hline $\mathrm{Fe} 4$ & -1.1622555927 & -0.0680175937 & -2.0574845815 \\
\hline $\mathrm{O} 5$ & -0.4773773402 & 0.2721723939 & -0.0245337343 \\
\hline H19 & -0.0197998813 & 1.1195827942 & -0.2373498981 \\
\hline $\mathrm{H} 20$ & -0.9121196573 & 0.2754788917 & 0.8419678617 \\
\hline O9 & -3.2069017891 & 0.3054045129 & -1.6946044387 \\
\hline $\mathrm{H} 23$ & -3.9243495893 & 0.0649971299 & -1.0919904700 \\
\hline
\end{tabular}




\begin{tabular}{|c|c|c|c|}
\hline H11 & -3.4851586737 & 0.5599383102 & -2.6048014572 \\
\hline $\mathrm{C} 1$ & 0.4730157885 & 1.9902243702 & -3.1855779800 \\
\hline $\mathrm{C} 3$ & -0.0656901050 & 3.0697801899 & -4.1047778962 \\
\hline $\mathrm{O} 4$ & -0.1840267605 & 1.7659215797 & -2.0719341901 \\
\hline O16 & 1.4376358294 & 1.2651186962 & -3.5768053027 \\
\hline O6 & 0.2189023788 & 4.3719950158 & -3.5274286290 \\
\hline H17 & 0.4206992235 & 2.9654748343 & -5.0755107362 \\
\hline $\mathrm{C} 8$ & -1.5954990426 & 2.9227511365 & -4.2872196458 \\
\hline C9 & -2.0158122284 & 1.5528200241 & -4.8450934683 \\
\hline $\mathrm{H} 21$ & -2.0597191075 & 3.0866891425 & -3.3184752945 \\
\hline O11 & -2.0231421899 & 3.9878224778 & -5.1797119088 \\
\hline $\mathrm{O} 12$ & -2.1214182042 & 0.5728996453 & -3.9639221601 \\
\hline $\mathrm{O} 13$ & -2.2477233008 & 1.4471339514 & -6.0673969992 \\
\hline H24 & -0.2777162296 & 5.0334969220 & -4.0550501423 \\
\hline $\mathrm{H} 25$ & -2.1656682464 & 3.5877255092 & -6.0674881565 \\
\hline $\mathrm{O} 25$ & 0.8189499681 & -0.9216095344 & -2.3833207100 \\
\hline $\mathrm{H} 26$ & 1.2971945788 & -0.1608816531 & -2.8787536239 \\
\hline $\mathrm{H} 27$ & 1.3287089378 & -1.2212996424 & -1.6126850432 \\
\hline \multicolumn{4}{|c|}{ catechol-Fe(II) } \\
\hline $\mathrm{Fe} 12$ & -0.5956779278 & -0.9401152716 & 0.2442911944 \\
\hline O19 & -0.0641533657 & -2.5860914580 & -0.8414605665 \\
\hline $\mathrm{H} 20$ & -0.4857873881 & -3.4434153186 & -0.9876671207 \\
\hline $\mathrm{H} 21$ & 0.7894117351 & -2.3994176083 & -1.4220580814 \\
\hline $\mathrm{O} 3$ & 0.9037483723 & 0.0994699490 & -0.5350751272 \\
\hline $\mathrm{O} 4$ & -1.1626559414 & 0.8549187687 & 0.8805537158 \\
\hline $\mathrm{O} 2$ & -1.9006279704 & -1.8627505236 & 1.5280222944 \\
\hline O9 & 1.9342327009 & -1.6494963794 & -1.9434301703 \\
\hline $\mathrm{H} 14$ & -1.8529718429 & -2.7250732998 & 1.9639493732 \\
\hline $\mathrm{H} 15$ & -2.4626456572 & -1.1440970974 & 2.0491894695 \\
\hline H16 & 1.7221785166 & -0.7547518909 & -1.4606774503 \\
\hline $\mathrm{H} 17$ & 2.2648531891 & -1.5603602027 & -2.8480258542 \\
\hline $\mathrm{C} 18$ & 1.7853262644 & 2.3837395314 & -0.6464735707 \\
\hline C19 & 1.6252613215 & 3.7306074347 & -0.2932518041 \\
\hline $\mathrm{C} 20$ & 0.5245932933 & 4.1333091482 & 0.4623212547 \\
\hline $\mathrm{C} 21$ & -0.4308054519 & 3.1944058586 & 0.8748758531 \\
\hline $\mathrm{C} 22$ & -0.2771226398 & 1.8582754859 & 0.5273418125 \\
\hline $\mathrm{C} 23$ & 0.8405206769 & 1.4491445309 & -0.2410467840 \\
\hline $\mathrm{H} 24$ & 2.6380529391 & 2.0553087099 & -1.2254254775 \\
\hline $\mathrm{H} 25$ & 2.3624061718 & 4.4568960240 & -0.6084718613 \\
\hline $\mathrm{H} 26$ & 0.4040237131 & 5.1732759769 & 0.7345625678 \\
\hline $\mathrm{H} 27$ & -1.2892951836 & 3.4918655486 & 1.4619238817 \\
\hline $\mathrm{O} 24$ & -2.8870026391 & 0.1722230671 & 2.5095915311 \\
\hline $\mathrm{H} 28$ & -2.2287624942 & 0.7235761957 & 1.9222535597 \\
\hline $\mathrm{H} 29$ & -3.7774477684 & 0.5429349855 & 2.5857212916 \\
\hline \multicolumn{4}{|c|}{ benzoquinone-Fe(II) } \\
\hline $\mathrm{O} 14$ & -2.1186606245 & -2.0330178699 & -3.4766712638 \\
\hline $\mathrm{H} 2$ & -1.7110437184 & -2.8065899684 & -3.8902478609 \\
\hline $\mathrm{Fe} 4$ & -1.5826270926 & -0.2725983695 & -2.9442561269 \\
\hline O5 & 0.4667181523 & -0.6707772548 & -2.4140302042 \\
\hline H19 & 0.7853925743 & -0.0436280888 & -1.6736822804 \\
\hline $\mathrm{H} 20$ & 0.7894363751 & -1.5789341100 & -2.3176158604 \\
\hline O9 & -1.7095024198 & 1.0633733797 & -1.5067325193 \\
\hline $\mathrm{H} 23$ & -2.5292589483 & 1.2951705630 & -1.0419301855 \\
\hline $\mathrm{C} 12$ & -0.9835245578 & 4.4261319780 & -4.2637500302 \\
\hline $\mathrm{C} 13$ & -0.7667867987 & 4.7969576318 & -5.6762862064 \\
\hline $\mathrm{C} 14$ & -0.7056628207 & 3.7041517454 & -6.6690358545 \\
\hline $\mathrm{C} 15$ & -0.8387391407 & 2.4191842623 & -6.2960011504 \\
\hline $\mathrm{C} 16$ & -1.0456754895 & 2.0722092741 & -4.8896318749 \\
\hline $\mathrm{C} 17$ & -1.1152442243 & 3.1409290226 & -3.8902286842 \\
\hline $\mathrm{H} 18$ & -1.0313787026 & 5.2453880592 & -3.5602276380 \\
\hline O19 & -0.6406081947 & 5.9946269726 & -6.0237298167 \\
\hline $\mathrm{H} 21$ & -0.5506789537 & 4.0018632805 & -7.6963036701 \\
\hline
\end{tabular}




\begin{tabular}{|c|c|c|c|}
\hline $\mathrm{H} 22$ & -0.8011329848 & 1.5959988243 & -6.9950094579 \\
\hline $\mathrm{O} 22$ & -1.1620190215 & 0.8568588095 & -4.5495591471 \\
\hline $\mathrm{H} 24$ & -1.2848007384 & 2.8159522541 & -2.8701704676 \\
\hline $\mathrm{O} 24$ & 0.6212912162 & 1.0513770642 & -0.5890245469 \\
\hline $\mathrm{H} 25$ & 1.1273764089 & 1.8511384761 & -0.3856808558 \\
\hline $\mathrm{H} 26$ & -0.3850810218 & 1.2049625036 & -0.7995724634 \\
\hline $\mathrm{O} 25$ & -3.8686768794 & -0.3105215563 & -3.3809351666 \\
\hline $\mathrm{H} 27$ & -3.6100572652 & -1.2646582916 & -3.5814909317 \\
\hline $\mathrm{H} 28$ & -4.6231026128 & -0.2362409792 & -2.7756407246 \\
\hline \multicolumn{4}{|c|}{ 1,4-naphthaquinone-Fe(II) } \\
\hline $\mathrm{O} 14$ & -2.3330166693 & -1.8529845874 & -3.1567442412 \\
\hline $\mathrm{H} 2$ & -2.1134553449 & -2.2013546529 & -4.0393784674 \\
\hline $\mathrm{Fe} 4$ & -2.0369847501 & 0.1127559584 & -2.7346519190 \\
\hline O5 & -0.0259184080 & -0.2820138265 & -2.0054277126 \\
\hline H19 & 0.1336151055 & 0.3770723073 & -1.3079220350 \\
\hline $\mathrm{H} 20$ & 0.0344234685 & -1.2316951577 & -1.6763840411 \\
\hline O9 & -2.0594151145 & 1.6108230847 & -1.5786382208 \\
\hline $\mathrm{H} 23$ & -2.6538379048 & 1.9814170321 & -0.9092494033 \\
\hline $\mathrm{C} 12$ & -0.6673207976 & 4.4013859133 & -4.4760857333 \\
\hline $\mathrm{C} 13$ & -0.3348266812 & 4.6574404834 & -5.8887552650 \\
\hline $\mathrm{C} 14$ & -0.4403463468 & 3.5185485271 & -6.8381550628 \\
\hline $\mathrm{C} 15$ & -0.8513348222 & 2.2488021337 & -6.3809740599 \\
\hline C16 & -1.1765864991 & 2.0606680104 & -4.9594305499 \\
\hline $\mathrm{C} 17$ & -1.0613474043 & 3.1926932120 & -4.0373574282 \\
\hline $\mathrm{H} 18$ & -0.5728363762 & 5.2499105422 & -3.8127930005 \\
\hline O19 & 0.0238390437 & 5.7978235123 & -6.2625896270 \\
\hline $\mathrm{O} 22$ & -1.5530482676 & 0.9283364746 & -4.5281508752 \\
\hline $\mathrm{H} 24$ & -1.3173729859 & 2.9720614262 & -3.0025330623 \\
\hline $\mathrm{O} 24$ & -0.6292734788 & -2.6999963722 & -1.4539537679 \\
\hline $\mathrm{H} 25$ & -0.4185315064 & -3.6185476096 & -1.2356206943 \\
\hline $\mathrm{H} 26$ & -1.4075003449 & -2.5671155202 & -2.1116253960 \\
\hline $\mathrm{O} 25$ & -4.2599455684 & -0.2228817903 & -3.0519551784 \\
\hline $\mathrm{H} 27$ & -4.0322538327 & -1.1904662335 & -3.1058509645 \\
\hline $\mathrm{H} 28$ & -5.0274303788 & -0.0117667436 & -2.4984258317 \\
\hline $\mathrm{C} 25$ & -0.1327384985 & 3.7016268505 & -8.1878418825 \\
\hline $\mathrm{C} 26$ & -0.2306508689 & 2.6345405221 & -9.0778808383 \\
\hline $\mathrm{C} 27$ & -0.6379922269 & 1.3753073624 & -8.6246998064 \\
\hline $\mathrm{C} 28$ & -0.9479634240 & 1.1824469747 & -7.2819352209 \\
\hline H17 & 0.1799122487 & 4.6816649576 & -8.5180291674 \\
\hline $\mathrm{H} 21$ & 0.0091713829 & 2.7807248456 & -10.1218902618 \\
\hline $\mathrm{H} 22$ & -0.7126979777 & 0.5497558889 & -9.3186721519 \\
\hline H32 & -1.2653547612 & 0.2183032713 & -6.9129865198 \\
\hline \multicolumn{4}{|c|}{ Edta-Fe(II) } \\
\hline $\mathrm{Fe} 1$ & -0.0201860345 & -0.0527985469 & -1.5477579672 \\
\hline $\mathrm{C} 2$ & 0.2309594348 & -0.5387212941 & 1.3284412618 \\
\hline $\mathrm{H} 3$ & 1.3159592907 & -0.4577066522 & 1.3206807399 \\
\hline $\mathrm{H} 4$ & -0.0443434899 & -0.9914672865 & 2.2802826269 \\
\hline $\mathrm{C} 5$ & -0.3938993231 & 0.8493336457 & 1.2016493685 \\
\hline H6 & -1.4768699862 & 0.7673251401 & 1.1280123340 \\
\hline $\mathrm{H} 7$ & -0.1686644532 & 1.4402745669 & 2.0897425281 \\
\hline N8 & 0.0656556284 & 1.5531936413 & -0.0457658184 \\
\hline N9 & -0.1465031056 & -1.4332481033 & 0.1815394047 \\
\hline $\mathrm{C} 10$ & -0.9429043415 & 2.5550688064 & -0.5162176409 \\
\hline H11 & -0.4554144080 & 3.2367788674 & -1.2128100505 \\
\hline H12 & -1.3490559029 & 3.1464925086 & 0.3044915698 \\
\hline $\mathrm{C} 13$ & -1.5210055282 & -1.9736284817 & 0.2507162620 \\
\hline H14 & -2.2347133009 & -1.1525255293 & 0.2993244005 \\
\hline $\mathrm{H} 15$ & -1.7405366469 & -2.4810626367 & -0.6890074449 \\
\hline $\mathrm{C} 16$ & 0.9102514866 & -2.4570427149 & -0.0983692907 \\
\hline H17 & 1.2861334043 & -2.9106441524 & 0.8142741908 \\
\hline $\mathrm{H} 18$ & 0.4726075719 & -3.2463783370 & -0.7102906298 \\
\hline
\end{tabular}




\begin{tabular}{|c|c|c|c|}
\hline C19 & 1.4523813934 & 2.1037980291 & 0.0260191245 \\
\hline $\mathrm{H} 20$ & 2.1315944497 & 1.3111421669 & 0.3268475968 \\
\hline H 21 & 1.7424284506 & 2.4260575356 & -0.9683609208 \\
\hline $\mathrm{C} 22$ & 2.0740081352 & -1.8402677262 & -0.8998822036 \\
\hline $\mathrm{O} 23$ & 3.2157526157 & -2.2986553661 & -0.8094911302 \\
\hline $\mathrm{O} 24$ & 1.7188718275 & -0.8098844195 & -1.6745545121 \\
\hline $\mathrm{C} 25$ & -1.8664813215 & -2.9477064727 & 1.3671694415 \\
\hline $\mathrm{O} 26$ & -2.9823940627 & -3.4263989896 & 1.5258391652 \\
\hline $\mathrm{O} 27$ & -0.8044216259 & -3.2552904155 & 2.1845071381 \\
\hline $\mathrm{C} 28$ & 1.5660572782 & 3.2549419054 & 1.0034869647 \\
\hline $\mathrm{O} 29$ & 1.4894827336 & 3.1540821300 & 2.2273547232 \\
\hline $\mathrm{O} 30$ & 1.7158020658 & 4.4588388207 & 0.3676049058 \\
\hline C31 & -2.0961154836 & 1.8544773266 & -1.2661276086 \\
\hline $\mathrm{O} 32$ & -3.2182232445 & 2.3628134285 & -1.3113295574 \\
\hline $\mathrm{O} 33$ & -1.7463647949 & 0.6997011022 & -1.8401363165 \\
\hline H34 & -1.0618301831 & -3.9012358449 & 2.8762685144 \\
\hline H41 & 1.7789520038 & 5.2025571125 & 1.0052252337 \\
\hline \multicolumn{4}{|c|}{ No Ligand-Fe(IV) } \\
\hline $\mathrm{O} 14$ & -0.7676618425 & -1.4982343393 & -1.9747904571 \\
\hline $\mathrm{H} 2$ & -1.4603922618 & -2.0492513920 & -2.3760190591 \\
\hline $\mathrm{Fe} 4$ & -0.3771310312 & 0.2612086252 & -2.4491642716 \\
\hline O5 & -1.2255465979 & -0.1137385897 & -4.3173038879 \\
\hline H19 & -0.9868767899 & 0.5176026774 & -5.0152567168 \\
\hline $\mathrm{H} 20$ & -2.2282784421 & -0.2120524595 & -4.1805713315 \\
\hline O9 & -1.8757872386 & 1.1643433113 & -1.7776676259 \\
\hline $\mathrm{H} 23$ & -1.8222603195 & 2.1370839914 & -1.7423046114 \\
\hline $\mathrm{O} 10$ & -3.5410971322 & -0.0349025390 & -3.3144509048 \\
\hline H11 & -4.3733520407 & -0.4848852251 & -3.1147829408 \\
\hline H12 & -3.1219901715 & 0.4726787970 & -2.5407150427 \\
\hline $\mathrm{O} 25$ & 1.0390217891 & -0.2117036898 & -1.0544518193 \\
\hline $\mathrm{H} 29$ & 0.8426441574 & -1.1446232389 & -0.8225357848 \\
\hline $\mathrm{H} 30$ & 1.9464288431 & 0.1041972450 & -0.9403174720 \\
\hline $\mathrm{O} 15$ & 0.4919303872 & 1.5436287290 & -3.0207902164 \\
\hline \multicolumn{4}{|c|}{ dma-Fe(IV) } \\
\hline O14 & 0.2674558505 & -1.3794106640 & -2.7588283622 \\
\hline $\mathrm{H} 2$ & 0.1159597623 & -2.3082724367 & -3.0138471775 \\
\hline $\mathrm{Fe} 4$ & -1.2238841767 & -0.1876842959 & -2.4887738774 \\
\hline O5 & 1.4084075656 & 0.2019226039 & -4.5505656020 \\
\hline H19 & 2.1370135086 & 0.7888971378 & -4.2909003747 \\
\hline $\mathrm{H} 20$ & 1.2014664353 & -0.4672458846 & -3.8292282519 \\
\hline O9 & -0.5383389718 & 1.4469013938 & -2.0067116754 \\
\hline $\mathrm{H} 23$ & -0.1823221579 & 1.6285030809 & -1.1181767825 \\
\hline $\mathrm{O} 15$ & -2.5597056719 & -1.1505109371 & -2.6038165346 \\
\hline $\mathrm{O} 10$ & -0.9454140708 & -0.7710294939 & -0.3933960884 \\
\hline H11 & -0.1440056731 & -1.3249520780 & -0.4926067915 \\
\hline H12 & -1.7005156098 & -1.2835516030 & -0.0522736931 \\
\hline H13 & -0.3820607680 & 0.8055453403 & -4.5552807397 \\
\hline N16 & -1.3718107850 & 0.5474139685 & -4.4011932344 \\
\hline $\mathrm{C} 17$ & -1.7473922921 & -0.4967530355 & -5.3897000424 \\
\hline $\mathrm{C} 18$ & -2.2584289754 & 1.7393835961 & -4.4411789683 \\
\hline $\mathrm{H} 22$ & -3.2757345509 & 1.4299441311 & -4.2068758849 \\
\hline $\mathrm{H} 24$ & -1.9116677037 & 2.4471868986 & -3.6941853312 \\
\hline $\mathrm{H} 25$ & -2.2412562320 & 2.1978102885 & -5.4333070991 \\
\hline $\mathrm{H} 26$ & -2.7099995782 & -0.9171316614 & -5.1114728369 \\
\hline $\mathrm{H} 27$ & -1.7920088278 & -0.0719732354 & -6.3955460585 \\
\hline $\mathrm{H} 28$ & -0.9956903584 & -1.2807216957 & -5.3663394575 \\
\hline \multicolumn{4}{|c|}{ tma-Fe(IV) } \\
\hline $\mathrm{O} 14$ & -0.1219741393 & -1.7468986180 & -3.2158580786 \\
\hline $\mathrm{H} 2$ & -0.6096047449 & -2.5871383628 & -3.2253702642 \\
\hline $\mathrm{Fe} 4$ & -0.9465209825 & -0.1360221249 & -2.7506010041 \\
\hline O5 & 1.1497702089 & 0.2124356110 & -2.8163068011 \\
\hline
\end{tabular}




\begin{tabular}{|c|c|c|c|}
\hline H19 & 1.7273383080 & 0.6408188564 & -2.1666872224 \\
\hline H20 & 1.3492389342 & -0.7462703168 & -2.9848872383 \\
\hline O9 & -1.1592430766 & 1.4118689302 & -1.7373747976 \\
\hline $\mathrm{H} 23$ & -0.4261571934 & 1.9885561648 & -1.4589095599 \\
\hline O15 & -2.4539037517 & -0.7254592138 & -2.5025316566 \\
\hline $\mathrm{O} 10$ & -3.8504478122 & 1.6793595182 & -1.5420423367 \\
\hline H11 & -4.0805065378 & 0.7329526296 & -1.5881716589 \\
\hline H12 & -2.8587845445 & 1.7255377424 & -1.4955221646 \\
\hline $\mathrm{C} 15$ & -0.1649511077 & 1.1252158610 & -5.3840875548 \\
\hline N16 & -1.4081500295 & 0.6494839274 & -4.7180376158 \\
\hline $\mathrm{C} 17$ & -2.0252329269 & -0.4604072149 & -5.5002952494 \\
\hline $\mathrm{C} 18$ & -2.3812211988 & 1.7714299073 & -4.5475343910 \\
\hline H17 & 0.2955054142 & 1.8971750752 & -4.7744694571 \\
\hline H18 & 0.5333063299 & 0.2976498904 & -5.4746687769 \\
\hline $\mathrm{H} 21$ & -0.3922057191 & 1.5274899372 & -6.3768099341 \\
\hline $\mathrm{H} 22$ & -3.2336070476 & 1.4330645256 & -3.9669507927 \\
\hline $\mathrm{H} 24$ & -1.9073726560 & 2.5677048271 & -3.9827798142 \\
\hline $\mathrm{H} 25$ & -2.7028040285 & 2.1409068078 & -5.5267923690 \\
\hline $\mathrm{H} 26$ & -2.9145295579 & -0.8000535808 & -4.9786163153 \\
\hline $\mathrm{H} 27$ & -2.2874137605 & -0.1176659393 & -6.5065740724 \\
\hline $\mathrm{H} 28$ & -1.3182667238 & -1.2837044734 & -5.5605251724 \\
\hline \multicolumn{4}{|c|}{ chloride-Fe(IV) } \\
\hline $\mathrm{O} 14$ & -0.2567421246 & -1.6753994051 & -2.4544211939 \\
\hline $\mathrm{H} 2$ & 0.4234916029 & -2.1870563155 & -2.9360707400 \\
\hline $\mathrm{Fe} 4$ & -0.2585410231 & 0.1073587212 & -2.6339383757 \\
\hline O5 & -1.4025090666 & 1.1067093371 & -3.9558461383 \\
\hline H19 & -1.0039047482 & 1.3825874610 & -4.7942221047 \\
\hline $\mathrm{H} 20$ & -2.3926097465 & 1.3177897201 & -3.8353063178 \\
\hline $\mathrm{Cl} 7$ & -2.0370420418 & 0.4222682937 & -1.0038266588 \\
\hline $\mathrm{O} 10$ & -3.7930373220 & 1.5064164494 & -3.2401795179 \\
\hline H11 & -4.6547677102 & 1.1984154573 & -3.5607950357 \\
\hline H12 & -3.6045148703 & 1.2226194088 & -2.3106170341 \\
\hline $\mathrm{O} 25$ & 0.6728210513 & 1.3911790114 & -1.3136725820 \\
\hline $\mathrm{H} 29$ & 1.5774761917 & 1.7226679869 & -1.4245130099 \\
\hline $\mathrm{H} 30$ & 0.2156806574 & 1.6496971350 & -0.4938924121 \\
\hline $\mathrm{O} 15$ & 1.0383441099 & 0.2068707632 & -3.6384647994 \\
\hline \multicolumn{4}{|c|}{ oxolate-Fe(IV) } \\
\hline $\mathrm{C} 1$ & -0.8326044840 & 3.8464913545 & -3.3168126623 \\
\hline $\mathrm{C} 2$ & -1.5379645714 & 2.6302145144 & -3.9087700508 \\
\hline $\mathrm{O} 3$ & -1.6246800787 & 0.1953742130 & -0.8615535043 \\
\hline $\mathrm{O} 4$ & -2.3150763713 & 1.9695621752 & -3.0876873066 \\
\hline O5 & -1.2526116725 & 2.2325784777 & -5.0773239562 \\
\hline O6 & -1.1058372143 & 5.0040765779 & -3.6018045093 \\
\hline O9 & -1.0515866981 & -1.9584668322 & -2.3840111876 \\
\hline $\mathrm{H} 10$ & -0.7824834896 & -2.2788578886 & -1.5055808062 \\
\hline H11 & -0.5711698928 & -2.3914120598 & -3.1098370371 \\
\hline $\mathrm{Fe} 12$ & -1.4848238163 & 0.1196158623 & -2.4832132691 \\
\hline $\mathrm{O} 13$ & 0.2470919775 & 0.8173180211 & -2.5862786070 \\
\hline $\mathrm{H} 14$ & 0.2594886869 & 2.5080330233 & -2.3143586270 \\
\hline H15 & 0.7384598900 & 0.5992527177 & -3.4044875255 \\
\hline O16 & -1.4117585753 & -0.2479259443 & -4.5262738027 \\
\hline H17 & -2.1532253612 & -0.7773840329 & -4.8686730954 \\
\hline $\mathrm{H} 18$ & -1.3589046776 & 0.6716775381 & -4.9974329570 \\
\hline $\mathrm{O} 17$ & 0.1370350613 & 3.5046483069 & -2.4302358273 \\
\hline $\mathrm{O} 18$ & -3.5400948776 & -0.2696288763 & -2.7589390177 \\
\hline H19 & -4.0433764569 & -0.6580468114 & -2.0228138324 \\
\hline $\mathrm{H} 20$ & -3.8384014829 & 0.6426104946 & -2.9789504957 \\
\hline \multicolumn{4}{|c|}{ malonate-Fe(IV) } \\
\hline $\mathrm{C} 1$ & -1.0169153310 & 1.3080309354 & -5.1643155328 \\
\hline $\mathrm{C} 2$ & -1.3739699689 & 2.6769038444 & -4.5472824056 \\
\hline $\mathrm{O} 3$ & -1.1950598136 & 0.2366433093 & -4.3711230525 \\
\hline $\mathrm{C} 4$ & -2.0669554798 & 2.4361975529 & -3.2358384413 \\
\hline
\end{tabular}

Page S27 of S31 


\begin{tabular}{|c|c|c|c|}
\hline H5 & -0.4479801240 & 3.2330343770 & -4.4064913840 \\
\hline O6 & -0.5884048921 & 1.2179720765 & -6.3186246903 \\
\hline $\mathrm{O} 14$ & -1.3552295741 & -1.9125338633 & -3.1069856091 \\
\hline $\mathrm{H} 2$ & -1.8545909338 & -2.5931746663 & -2.6296919226 \\
\hline H3 & -1.5797833179 & -1.8302019128 & -4.0564140206 \\
\hline $\mathrm{Fe} 4$ & -1.0948863774 & 0.0161832144 & -2.4310777849 \\
\hline O5 & 0.9447216702 & -0.1061917349 & -2.8237499204 \\
\hline H19 & 1.6158969561 & -0.2443484949 & -2.1378884597 \\
\hline $\mathrm{H} 20$ & 1.2763759724 & -0.1279179505 & -3.7362423652 \\
\hline O9 & -3.2696705630 & -0.0069822216 & -2.1251063657 \\
\hline $\mathrm{H} 23$ & -3.4485606306 & -0.1430629959 & -1.1775795201 \\
\hline H11 & -3.6324066402 & 0.8741827051 & -2.4178876229 \\
\hline $\mathrm{O} 15$ & -0.8661509385 & -0.3880427825 & -0.8551450215 \\
\hline $\mathrm{H} 21$ & -2.0140813211 & 3.2147358942 & -5.2398838509 \\
\hline $\mathrm{O} 21$ & -3.3076421273 & 2.5377957425 & -3.0990319894 \\
\hline $\mathrm{O} 23$ & -1.2846358028 & 1.9169988091 & -2.2742739389 \\
\hline \multicolumn{4}{|c|}{ tartrate-Fe(IV) } \\
\hline $\mathrm{O} 14$ & -1.8801956895 & -1.7716265730 & -2.8299685378 \\
\hline $\mathrm{H} 2$ & -1.6315618328 & -2.6364459058 & -2.4678596123 \\
\hline H3 & -1.8315928359 & -1.7052421369 & -3.8068413722 \\
\hline $\mathrm{Fe} 4$ & -1.1220764282 & 0.0374152509 & -2.1625962285 \\
\hline O5 & 0.7973379774 & -0.7906793194 & -2.6177268308 \\
\hline H19 & 1.3377312942 & -0.0396253888 & -3.0248419096 \\
\hline $\mathrm{H} 20$ & 1.2431597112 & -1.1520996266 & -1.8319777290 \\
\hline O9 & -3.0737704682 & 0.7169229333 & -1.8290321371 \\
\hline $\mathrm{H} 23$ & -3.4017891893 & 0.7931860220 & -0.9182440068 \\
\hline H11 & -3.7719962682 & 0.7000856463 & -2.5028347961 \\
\hline $\mathrm{O} 15$ & -0.9959497283 & -0.4214955489 & -0.5944910721 \\
\hline $\mathrm{C} 1$ & 0.4705178640 & 2.1322604441 & -3.1307995517 \\
\hline $\mathrm{C} 3$ & -0.1703559743 & 3.1981177818 & -3.9976274555 \\
\hline $\mathrm{O} 4$ & -0.2382817293 & 1.7433499405 & -2.0783573587 \\
\hline O16 & 1.5201339039 & 1.5510432504 & -3.5017462528 \\
\hline O6 & -0.1492938366 & 4.4762590210 & -3.3194029374 \\
\hline H17 & 0.3773252730 & 3.2456446349 & -4.9393791956 \\
\hline $\mathrm{C} 8$ & -1.6385096893 & 2.7863906783 & -4.2706634896 \\
\hline C9 & -1.7650436030 & 1.3776720906 & -4.8684928553 \\
\hline $\mathrm{H} 21$ & -2.1606536056 & 2.8257563865 & -3.3172677229 \\
\hline $\mathrm{O} 11$ & -2.2210632654 & 3.7749793297 & -5.1519207094 \\
\hline $\mathrm{O} 12$ & -1.5140426455 & 0.3534024263 & -4.0607964966 \\
\hline $\mathrm{O} 13$ & -2.1067380684 & 1.2436720694 & -6.0555170603 \\
\hline $\mathrm{H} 24$ & -0.6681356974 & 5.1103850272 & -3.8583643017 \\
\hline $\mathrm{H} 25$ & -2.3413207967 & 3.3707520791 & -6.0402548917 \\
\hline \multicolumn{4}{|c|}{ catechol-Fe(IV) } \\
\hline $\mathrm{Fe} 12$ & -0.8043398489 & -0.7618731585 & 0.1663471937 \\
\hline O19 & 0.0945217808 & -2.5595601633 & -0.1652404955 \\
\hline $\mathrm{H} 20$ & 1.0451791380 & -2.4446699329 & -0.3454811913 \\
\hline $\mathrm{H} 21$ & -0.3397245506 & -3.2500927179 & -0.6890240956 \\
\hline $\mathrm{O} 14$ & -2.3542943879 & -1.3319345919 & 0.0538900022 \\
\hline $\mathrm{O} 3$ & 1.0412266998 & -0.1896954464 & 0.2247675633 \\
\hline $\mathrm{O} 4$ & -1.2219017022 & 1.0715491300 & 0.3032417816 \\
\hline $\mathrm{O} 2$ & -0.6671904426 & -1.1013158427 & 2.2635509843 \\
\hline O9 & -0.6909098452 & -0.5498117682 & -2.0460772650 \\
\hline H14 & 0.0638823690 & -0.6694127106 & 2.7361109979 \\
\hline $\mathrm{H} 15$ & -1.4989368126 & -1.1237311354 & 2.7650912929 \\
\hline $\mathrm{H} 16$ & -0.0561709325 & 0.1412880660 & -2.3084304602 \\
\hline H17 & -1.5924174104 & -0.3469216281 & -2.3567102223 \\
\hline $\mathrm{C} 18$ & 2.3398816848 & 1.8924428083 & 0.3375312608 \\
\hline C19 & 2.3003314316 & 3.2896680716 & 0.4187708728 \\
\hline $\mathrm{C} 20$ & 1.0773503832 & 3.9661067881 & 0.4637561504 \\
\hline $\mathrm{C} 21$ & -0.1278488538 & 3.2560991196 & 0.4275607196 \\
\hline $\mathrm{C} 22$ & -0.0919328082 & 1.8678954588 & 0.3472582401 \\
\hline $\mathrm{C} 23$ & 1.1437141201 & 1.1831560071 & 0.3024621724 \\
\hline
\end{tabular}




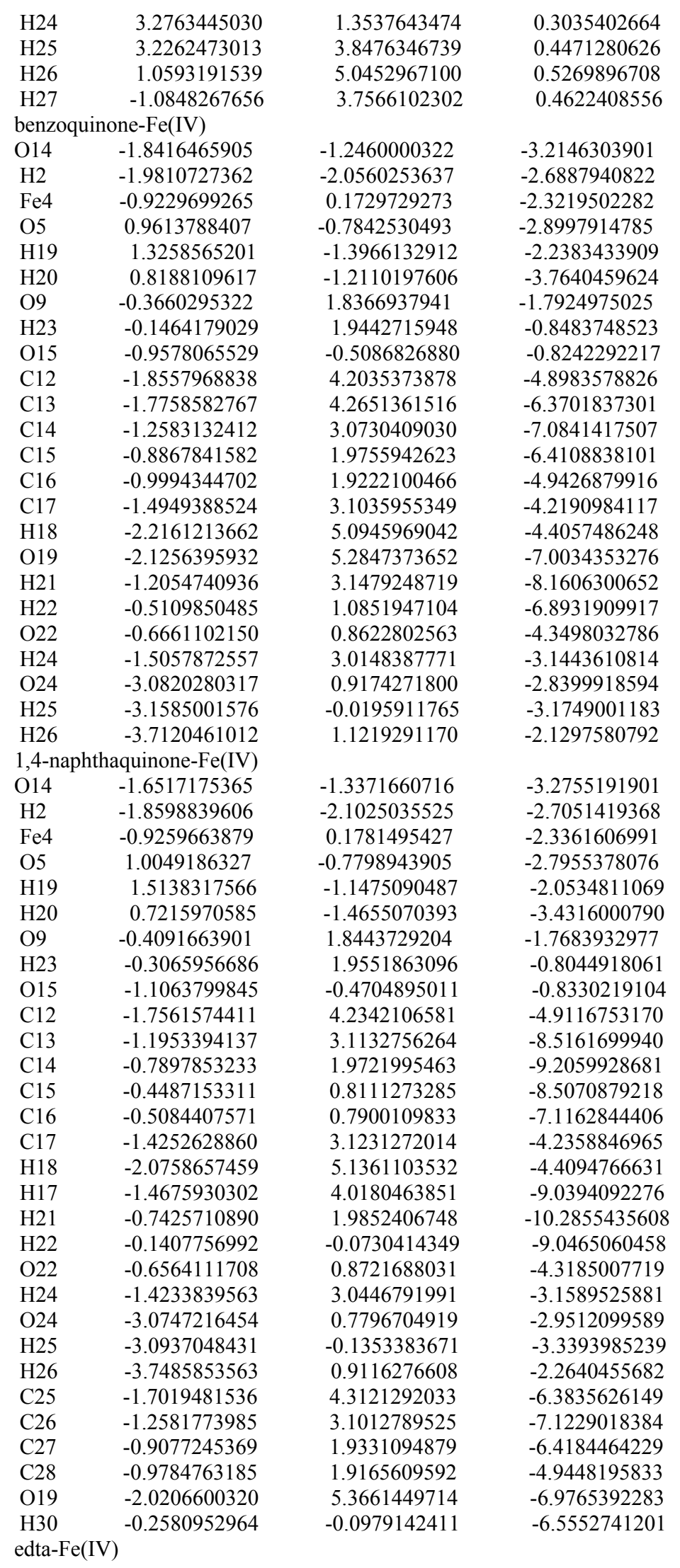




$\begin{array}{lrrr}\text { Fe1 } & -0.1603003512 & -0.1022444073 & -1.3974872379 \\ \text { C2 } & 0.4466970058 & -0.4559928965 & 1.4506417070 \\ \text { H3 } & 1.4800091851 & -0.1825581253 & 1.2558330994 \\ \text { H4 } & 0.4025673588 & -0.9295879976 & 2.4303778493 \\ \text { C5 } & -0.4283897782 & 0.7940037394 & 1.4346118295 \\ \text { H6 } & -1.4803835430 & 0.5305103584 & 1.4991049860 \\ \text { H7 } & -0.1974096335 & 1.4138130166 & 2.2942967777 \\ \text { N8 } & -0.2573856866 & 1.5671524965 & 0.1502770960 \\ \text { N9 } & 0.0365044162 & -1.4045586258 & 0.3725860405 \\ \text { C10 } & -1.4434040114 & 2.3827221038 & -0.2387364167 \\ \text { H11 } & -2.2943007975 & 1.7050620670 & -0.3094218074 \\ \text { H12 } & -1.2766535251 & 2.7523600504 & -1.2449683142 \\ \text { C13 } & -1.2681303540 & -2.0761317153 & 0.5873053527 \\ \text { H14 } & -2.0580306549 & -1.3342159357 & 0.6494466263 \\ \text { H15 } & -1.5071850389 & -2.6389700215 & -0.3143490334 \\ \text { C16 } & 1.0851496829 & -2.3673177686 & -0.0764890173 \\ \text { H17 } & 2.0530581426 & -1.8831258971 & 0.0191076008 \\ \text { H18 } & 1.0937263964 & -3.2745326999 & 0.5202130397 \\ \text { C19 } & 1.0204509900 & 2.3519064989 & 0.0619310401 \\ \text { H20 } & 1.8367166106 & 1.6415963744 & -0.0325268122 \\ \text { H21 } & 0.9909936577 & 2.9126895659 & -0.8644892977 \\ \text { C22 } & 0.8791892170 & -2.7168118181 & -1.5613762928 \\ \text { O23 } & 1.3863765871 & -3.7367931511 & -2.0451615212 \\ \text { O24 } & 0.1505319306 & -1.8323229770 & -2.2415436738 \\ \text { C25 } & -1.4156316478 & -3.0070005110 & 1.7749270916 \\ \text { O26 } & -2.4759777929 & -3.5369092388 & 2.0884745070 \\ \text { O27 } & -0.2572762715 & -3.2162165207 & 2.4890097403 \\ \text { C28 } & 1.2905829470 & 3.2790720496 & 1.2223116671 \\ \text { O29 } & 1.6739108447 & 2.9352514084 & 2.3387956134 \\ \text { O30 } & 1.0906318743 & 4.5983214088 & 0.8892396302 \\ \text { C31 } & -1.8414745162 & 3.5633776268 & 0.6192772333 \\ \text { O32 } & -2.4135527693 & 4.5605897196 & 0.1957896035 \\ \text { O33 } & -1.5588226995 & 3.3916397658 & 1.9554541418 \\ \text { H34 } & -0.4085316466 & -3.8442180320 & 3.2269303750 \\ \text { O35 } & -0.1573087997 & 1.2691854094 & -3.0059876856 \\ \text { H36 } & -0.8532358650 & 1.2267850911 & -3.6840370991 \\ \text { H37 } & 0.7475586686 & 1.3649587759 & -3.3560842194 \\ \text { O38 } & -1.8196253529 & -0.1454599430 & -1.4163787988 \\ \text { H41 } & 1.3057011566 & 5.1921796455 & 1.6400648647 \\ \text { H40 } & -1.8655126093 & 4.1581211449 & 2.4840582014 \\ \text { O41 } & 1.7105668011 & 0.2230088512 & -1.4632438641 \\ \text { H42 } & 2.2790909087 & -0.4051840608 & -1.9457193572\end{array}$

List S1. Coordinates of DFT-optimized structures 


\section{References}

(1) Colussi, A.; Enami, S., Detecting Intermediates and Products of Fast Heterogeneous Reactions on Liquid Surfaces via Online Mass Spectrometry. Atmosphere 2019, 10, (2).

(2) Enami, S.; Mishra, H.; Hoffmann, M. R.; Colussi, A. J., Hofmeister effects in micromolar electrolyte solutions. J Chem Phys 2012, 136, (15), 154707.

(3) Enami, S.; Colussi, A. J., Long-range specific ion-ion interactions in hydrogen-bonded liquid films. Journal of Chemical Physics 2013, 138, (18).

(4) Enami, S.; Hoffmann, M. R.; Colussi, A. J., How phenol and alpha-tocopherol react with ambient ozone at gas/liquid interfaces. J Phys Chem A 2009, 113, (25), 7002-10.

(5) Kebarle, P.; Verkerk, U. H., Electrospray: from ions in solution to ions in the gas phase, what we know now. Mass Spectrom Rev 2009, 28, (6), 898-917.

(6) Xiong, H.; Lee, J. K.; Zare, R. N.; Min, W., Strong Electric Field Observed at the Interface of Aqueous Microdroplets. J Phys Chem Lett 2020, 11, (17), 7423-7428.

(7) Enami, S.; Hoffmann, M. R.; Colussi, A. J., Proton Availability at the Air/Water Interface. The Journal of Physical Chemistry Letters 2010, 1, (10), 1599-1604.

(8) Levanov, A. V.; Isaikina, O. Y.; Lunin, V. V., Thermodynamic and Kinetic Parameters of the Solubility of Ozone in Water. Russian Journal of Physical Chemistry A 2019, 93, (7), 1230-1234.

(9) Chou, M.-S.; Chang, K.-L., Oxidation of Aqueous Dimethyl Sulfoxide (DMSO) Using UV, O3, and UV/O3. Ozone: Science \& Engineering 2007, 29, (5), 391-397. 